\title{
Immunomodulation by radiotherapy in tumour control and normal tissue toxicity
}

DOI:

10.1038/s41577-021-00568-1

\section{Document Version}

Accepted author manuscript

Link to publication record in Manchester Research Explorer

\section{Citation for published version (APA):}

Cytlak, U. M., Dyer, D. P., Honeychurch, J., Williams, K. J., Travis, M. A., \& Illidge, T. M. (2021).

Immunomodulation by radiotherapy in tumour control and normal tissue toxicity. Nature Reviews Immunology. https://doi.org/10.1038/s41577-021-00568-1

\section{Published in:}

Nature Reviews Immunology

\section{Citing this paper}

Please note that where the full-text provided on Manchester Research Explorer is the Author Accepted Manuscript or Proof version this may differ from the final Published version. If citing, it is advised that you check and use the publisher's definitive version.

\section{General rights}

Copyright and moral rights for the publications made accessible in the Research Explorer are retained by the authors and/or other copyright owners and it is a condition of accessing publications that users recognise and abide by the legal requirements associated with these rights.

\section{Takedown policy}

If you believe that this document breaches copyright please refer to the University of Manchester's Takedown Procedures [http://man.ac.uk/04Y6Bo] or contact uml.scholarlycommunications@manchester.ac.uk providing relevant details, so we can investigate your claim.

\section{OPEN ACCESS}


Title: Immunomodulation by radiotherapy in tumour control and normal tissue toxicity.

Authors: Urszula M Cytlak ${ }^{1,2^{*}}$, Douglas P Dyer ${ }^{1}$, Jamie Honeychurch ${ }^{2}$, Kaye J Williams ${ }^{3}$, Mark A Travis ${ }^{1 *}$ and Timothy M Illidge ${ }^{2 *}$

Affiliations: ${ }^{1}$ Lydia Becker Institute for Immunology and Inflammation, Wellcome Centre for Cell-Matrix Research, Manchester Collaborative Centre for Inflammation Research, Faculty of Biology, Medicine and Health, University of Manchester, Manchester, UK; ${ }^{2}$ Targeted Therapy Group, Division of Cancer Sciences, Cancer Research UK Manchester Institute, University of Manchester, Manchester, UK; ${ }^{3}$ Division of Pharmacy and Optometry, Faculty of Biology, Medicine and Health, University of Manchester, Manchester, UK.

\section{Keywords}

Radiotherapy, normal tissue toxicity, chronic inflammation, immune modulation, TGF $\beta$ signalling, cytokines/chemokines, extracellular matrix

\section{*Corresponding authors:}

Urszula M Cytlak: urszula.cytlak-chaudhuri@manchester.ac.uk

Mark A Travis: mark.travis@manchester.ac.uk

Timothy M Illidge: tim.illidge@manchester.ac.uk 


\begin{abstract}
Radiotherapy (RT) is a highly effective anti-cancer treatment that is delivered to over half of all cancer patients. In addition to the well documented direct cytotoxic effects, RT can induce immunomodulatory effects to the tumour and surrounding tissues. These effects are thought to underlie the so-called 'abscopal responses', where RT generates systemic antitumour immunity outside of the irradiated tumour. In addition, RT may also result in an inflammatory immune response in the normal tissues surrounding the tumour. The full scope of these immune changes remains unclear but is likely to involve multiple tissue components and immune mediators. These include immune cells, the extracellular matrix, endothelial and epithelial cells and a myriad of chemokines and cytokines, including TGF $\beta$, which is known to play a key role. In normal tissues exposed to RT during cancer therapy, acute immune changes may ultimately lead to chronic inflammation and RT-induced toxicity and organ dysfunction, which limits the quality of life of cancer survivors. Here, we discuss the emerging understanding of RT-induced immune effects with a particular focus on the lung and gut and the potential immune cross-talk that occurs between these tissues.
\end{abstract}




\section{Introduction}

Radiotherapy (RT) is an important component of anti-cancer management and is delivered to around $50-60 \%$ of all cancer patients, with $40 \%$ of those cured of cancer having received RT. In the last century the focus was on the direct cytotoxic effects of RT on cancer cells to induce irreparable DNA damage. More recently the immunomodulatory effects of RT on both the tumour microenvironment (TME) as well as on the surrounding normal tissue are increasingly recognised ${ }^{1-3}$. The effects of RT on the immune system within the irradiated tumour can be either immunostimulatory or immunosuppressive, which is likely to be influenced by the immune contexture and dose and fractionation of $\mathrm{RT}^{4}$. Local $\mathrm{RT}$ may also promote systemic anti-tumour immunity, so-called 'abscopal responses', leading to the control of secondary metastases ${ }^{5}$. However, in the clinic such systemic responses are rare ${ }^{6}$, presumably secondary to the dominant immunosuppressive TME or insufficient escalation of anti-tumour responses. In addition to the RT-induced tumour effects, local RT may also lead to acute and late toxicity in surrounding normal tissue, that may also extend to distal sites outside of the irradiated field ${ }^{7-9}$. Given the recent major breakthrough of the immune checkpoint inhibitors, with anti-CTLA-4 and anti-PD-1/PD-L1 monoclonal antibodies (mAb) leading to durable remissions in some incurable cancers ${ }^{10}$, there has been intense recent interest in trying to further increase the immunostimulatory effects of RT in combination with immune checkpoint blockade or other immunomodulating agents, and to promote enhanced systemic immunity to secondary tumours ${ }^{4,11-13}$.

The ability of RT to result in local tumour control is well established and integrated into routine management of many cancer types. However, despite recent technological advances in delivery ${ }^{7,14}$ that minimise exposure of surrounding healthy normal tissues, RT-induced normal tissue toxicity remains a major limitation to improving overall cancer outcomes and a significant cause of decreased quality of life of cancer survivors ${ }^{6,8}$. To date, RT-induced immunological responses underlying the acute and late-stage toxicity in normal tissue are poorly understood. In this Review, we will focus on RT-induced immune changes within tumours and the surrounding normal tissue. We will specifically discuss the RT-induced immunological effects in healthy gut and lung tissue, given that these mucosal barrier sites are the most commonly exposed normal tissues for thoracic, abdominal and pelvic cancers treated with RT, resulting in frequent and disabling acute and late toxicity ${ }^{7,9,15-17}$. 


\section{Immunomodulatory effects of radiotherapy}

RT can cause numerous changes to the tissue environment, including induction of cell death, alterations in cellular metabolism and local tissue damage to which inflammatory responses are rapidly escalated. These effects can be directly induced by RT, or indirectly mediated by the immune system which detect the release of alarmins and damage-associated molecular patterns (DAMPs) upon RT-mediated cell death ${ }^{18,19}$. Such inflammatory responses to irradiation have some similarities to those normally mounted in the presence of a pathogen but in contrast lead to the induction of sterile inflammation ${ }^{20}$.

Thus, following RT-induced cell death, cells release their DNA and RNA which can be sensed by immune cells via a multitude of nucleic acid recognition molecules and pathways ${ }^{21}$. Of these, the cyclic GMP-AMP synthase (cGAS)-stimulator of interferon genes (STING) pathway, which leads to activation of interferon (IFN) responses is considered of key importance to the propagation of RT-induced immune responses leading to tumour control $^{5,22}$. RT also leads to the generation of reactive oxygen species (ROS) and reactive nitrogen species (RNS) that are able to attract and activate immune effector cells of the innate immune system. These include neutrophils and natural killer (NK) cells, that are triggered to mount phagocytic and cytotoxic responses ${ }^{23-25}$, clearing tissue debris and damaged cells and preparing for potential further insult. Additionally, cells in the process of RT-induced cell death can upregulate a spectrum of $\mathrm{DAMPs}^{26}$ that recruit and enhance dendritic cell (DC ${ }^{23}$ activation and antigen processing, which can promote activation of both $\mathrm{CD} 4^{+}$and $\mathrm{CD} 8^{+} \mathrm{T}$ cell responses. RT is known to also activate monocytes and tissue macrophages which can be polarised to either M1-like or M2-like macrophage phenotypes ${ }^{18,27,28}$.

Moreover, RT-induced effects on the immune system can be propagated via a plethora of cytokines that have been reported to accumulate in irradiated tissues ${ }^{29-32}$. Such cytokines include interferon gamma (IFN $\gamma$ ), interleukin (IL)-1ß, IL-3, IL-4, IL-6, tumour necrosis factor (TNF) and transforming growth factor beta (TGF $\beta$ ). Furthermore, increased levels of the chemokine ligands CCL2, CCL3, CCL5, CXCL9, CXCL10, and CXCL16 have also been reported in a range of tissues after $\mathrm{RT}^{33-36}$. The specific $\mathrm{RT}$-related function of these inflammatory mediators is increasingly realised to be tissue-dependent, with contributions from other components of the tissue and cancer cells ${ }^{2,29,30,37,38}$. 
The initial effects of RT on the immune system are in part dictated by the apparent differential cytotoxic effect on immune effector cells, with lymphocytes known to be more sensitive to RT-induced cell death than myeloid cell subsets ${ }^{39}$. RT-induced DNA damage in highly proliferating cells may underlie the higher radiosensitivity of circulating lymphocytes, with RT inducing lymphocyte apoptosis during the interphase ${ }^{40}$. Within the lymphocyte population, B cells were found to be the most sensitive to RT-induced death, while T cell subsets display a spectrum of sensitivity that appears to be correlated with the efficiency of DNA repair mechanisms or their activation status ${ }^{41}$. Resting $T$ cells were relatively radiosensitive, with $T$ cells stimulated with anti-CD3/anti-CD28 showing more resistance to RT compared with unstimulated and non-proliferating $T$ cells ${ }^{41}$. This relative protection from $\mathrm{RT}$ in activated T cells was associated with reduced expression and less phosphorylation of ataxia-telangiectasia mutated (ATM) kinase $^{41}$. Therefore, ATM and its targets were considered to be key in promoting responses to ionizing radiation as the major DNA damage response activated by double-stranded DNA (dsDNA) breaks ${ }^{41}$. Interestingly, patients with increased ATM expression may have altered radiosensitivity ${ }^{7}$.

The pattern of RT sensitivity within $\mathrm{T}$ cell subsets has been investigated in multiple experimental and irradiation settings ${ }^{39}$ with a degree of conflicting results. Some studies report that memory $\mathrm{T}$ cells and $\mathrm{T}$ cells within the tumour or associated tissue are able to withstand more irradiation than naïve and circulating $\mathrm{T}$ cells $\mathrm{s}^{42,43}$. Overall, $\mathrm{CD} 4^{+} \mathrm{T}$ cells are considered more radioresistant than $\mathrm{CD}^{+} \mathrm{T}$ cells, with regulatory $\mathrm{T}$ cells (Treg) even more resistant to $\mathrm{RT}^{39,43,44}$. While this enhanced resistance is associated with better DNA and oxidative repair mechanisms, the levels of certain immune mediators and cytokines such as TGF $\beta$ have also been shown to contribute to the radioresistance of tumour-infiltrating $T$ cells $s^{42}$.

Myeloid-derived cells such as monocytes and DCs are relatively more radioresistant than lymphocytes and NK cells, with macrophages and granulocytes showing even higher levels of resistance to radiation-induced death ${ }^{39,45,46}$. This enhanced radioresistance is likely due to myeloid cells not actively proliferating to the same extent as lymphocytes, and thus are less prone to RT-induced apoptosis. Interestingly, within the macrophage population, M2-like macrophages were found to be more resistant to RT than M1-like macrophages in an in vitro 
assay ${ }^{47}$, potentially contributing to the observed accumulation of M2-like macrophages within irradiated tumours and normal tissues following $\mathrm{RT}^{27,28,37}$.

Interestingly, differences in the radiosensitivity of human immune cells are observed between individuals, with sex- and age-related differences reported ${ }^{39}$. These differences in radiosensitivity are likely to be driven by additional mechanisms well beyond proliferative capacity and is an important and underexplored research area requiring further investigation. Thus, RT can have a variety of effects on immune cell populations, both directly and indirectly via changes to the tissue microenvironment. Differential sensitivity of immune cells to RT raises the possibility that certain subsets will be preferentially ablated following treatment. Given that lymphocytes are highly sensitive, it is possible that RT may lead to depletion of tumour-specific T cells and that the extent to which this occurs may be influenced by the dose and particularly number of fractions delivered over time. It is therefore possible that RT may bias the immune contexture towards more resistant populations, which are generally considered to be immunosuppressive ${ }^{37}$. However, there is likely a balance here between direct cytotoxic effects and the immunostimulatory effects of RT that drive T cell priming and activation. It is clear from preclinical models that RT can enhance T cell expansion within the $\mathrm{TME}^{42}$, although the RT fractionation regimen employed may be key, as most reports use single or a small number of fractions of RT. Understanding the impact and mechanistic basis of RT on the immune repertoire is important in enhancing future therapies aimed to stimulate immune responses in combination with RT.

\section{Immunological effects of RT on tumours}

RT has been shown to upregulate several cell surface molecules on tumour cells including MHC-I ${ }^{3}, \mathrm{CD} 80, \mathrm{CD}^{23} 6^{23}$, the apoptosis-inducing death receptor FAS (also known as CD95), several NK group 2D (NKG2D) ligands and the intercellular adhesion molecule ICAM1 ${ }^{48}$. Upregulation of these molecules can enhance recognition of cancer cells by immune cells and promote more effective activation of anti-tumour immune responses. The release of dsDNA from dying irradiated tumour cells, activation of the cGAS-STING pathway and type I IFN production by tumour cells and DCs appears key in propagation of effective RT-induced antitumour responses ${ }^{22}$. STING-dependent IFN $\beta$ production was associated with better recruitment of DCs and more efficient cross-priming of tumour antigens to $\mathrm{CD} 8^{+} \mathrm{T}$ cells in 
irradiated tumours, which was also critical for better protection from distal metastases ${ }^{5,22}$. Thus, dsDNA is a key DAMP that enhances RT-induced immunogenic cell death ${ }^{5,22}$.

Following RT-induced cellular stress and ROS release, several other DAMPs - including ATP, high mobility group box 1 (HMGB1) and calreticulin (CRT) - are released by many tumour cells ${ }^{49-51}$. Via receptor-mediated interaction, these DAMPs accentuate more efficient immunogenic cell death following RT: ATP-P2X7 purinergic receptor (P2X7R) signalling enhanced immune cells recruitment, CRT-CD91 interaction enhanced phagocytosis and HMGB1-TLR4 signalling led to better processing and presentation of tumour antigens by DCs ${ }^{52,53}$. Additionally, RT may potentially liberate novel tumour-specific antigens (resulting from the high mutation rate in cancer cells) and thus enhance non-self-recognition by $T$ cells $s^{3,54}$.

The RT-induced immunomodulatory changes on tumour cells, together with the RTinduced activation of immune cells and effects on other cellular and non-cellular tissue components, contribute to the production of pro-inflammatory cytokines and chemokine homing signals that enhance influx of effector immune cells into the TME, promoting antitumour responses ${ }^{12,23,37,50}$. Increased levels of DC-derived IL-1 $\beta$ were linked to the release of ATP from dying tumour cells, which acted on P2X7Rs expressed by DCs and stimulated the NLRP3-dependent inflammasome ${ }^{53}$. Resultant IL-1 $\beta$ secretion enhanced CCL2 production, and increased $\mathrm{CCR}^{+}$monocyte and $\mathrm{T}$ cell recruitment ${ }^{33,55}$, promoting the further development of a pro-inflammatory microenvironment via production of TNF and IFN $\gamma^{37}$. These pro-inflammatory cytokines have been reported to persist in irradiated tissues and in the blood of cancer patients even several weeks after $\mathrm{RT}^{32,37}$. However, efficient infiltration of effector immune cells into tumours might be hindered by the aberrant tumour vasculature and hypoxic regions that are known to exist within the TME of many solid tumours and promote an immunosuppressive environment ${ }^{37,56}$. Due to the immunostimulatory effects of RT on the surrounding tissues and vasculature ${ }^{2}$, low doses of RT may promote gradual reoxygenation of tumours and thus enhance influx of anti-tumour immune effector cells $s^{57}$.

In contrast to these immunostimulatory effects, RT can also result in immunosuppressive consequences as outlined earlier, thought to dependent on the immune contexture and RT dose and fractionation ${ }^{4}$ (Box 1). RT and associated ROS can enhance generation and activation of TGF $\beta$ from multiple immune cells, tissue components and TME-associated cells ${ }^{58}$. In turn, 
TGF $\beta$ can enhance polarisation of macrophages to an M2-like and tumour-associated macrophage phenotype ${ }^{27}$, that is associated with the secretion of anti-inflammatory and profibrogenic cytokines. Enhanced polarisation of Treg cells, differentiation of cancer-associated fibroblasts (CAFs) and recruitment of tumour-associated myeloid-derived suppressor cells (MDSCs) can also be driven by TGF $\beta^{32,59-61}$. Additionally, recruitment of CCR2 ${ }^{+}$Ly6 $\mathrm{C}^{\text {hi }}$ MDSCs into irradiated tumours was dependent on RT-induced STING-IFN I pathway activation ${ }^{62}$. CAFs promote recruitment and survival of more Treg cells by secreting CXCL12, and their retention in the TME via OX4OL and PD-L2 binding ${ }^{60}$.

Some of these immunosuppressive effects are driven by the tumour-associated suppressor cells and RT may accelerate further expansion of these populations ${ }^{37,60}$. Furthermore, the enhanced radiosensitivity of immune effector cell populations versus cancer and cancerassociated cells may also contribute to an immunosuppressive environment ${ }^{44}$. Moreover, tumour cells can also evade immune detection through the upregulation of immune checkpoint receptors (for example PD-L1 and CTLA-4) and RT can enhance the expression of immune checkpoint receptors on both cancer and immune cells ${ }^{25,63}$.

In summary, RT may induce an immunostimulatory environment potentially enhancing tumour control but may also enhance the recruitment and phenotypic change of immunosuppressive cells within the TME that abrogate effective anti-tumour immune responses. The underlying factors that dictate the overall immunological consequences of RT are likely to be multifactorial and tumour-dependent (e.g. tumour mutational burden), organdependent (e.g. skin, gut versus breast, prostate), and RT delivery-dependent (e.g. dose per fraction, total dose, time between fractions and overall treatment time).

\section{Acute immunological effects of RT on normal tissue}

Whilst the immunomodulatory effects of RT on the TME appear important for tumour control, the effects on the surrounding normal tissues are also critical and are frequently dose limiting for RT, leading to both acute and late side effects.

Several components of the TME and surrounding normal healthy tissue included in the RT clinical target volume (CTV) may contribute to the observed elevation in inflammatory signalling following RT, including changes to the vascular and epithelial cells (Box 2). Alterations in chemokine expression appears key in promoting immune cell recruitment to 
the irradiated CTV including normal tissues adjacent to RT-treated tumour sites ${ }^{64}$. For example, recruitment of effector T cells was found to be promoted by CXCL16 ${ }^{35}$, while monocytes and neutrophils were attracted by increased levels of CCL2, CCL5 and CCL3 $33,34,36$. Macrophages can generate CXCL9 and CXCL10 and further promote effector T cell recruitment ${ }^{38}$. Within hours of RT exposure, infiltrating inflammatory immune cells can act to protect the normal tissues after RT-induced breakdown of endothelial and epithelial barriers ${ }^{2}$ (Figure 1). In the next stages, the accumulating immune cells can induce further inflammation by secretion of pro-inflammatory cytokines, for example an increase in TNF and IL-6 that can trigger endothelial cell activation after RT exposure and escalation of the immune cell recruitment ${ }^{65}$. RT can also enhance the expression of certain adhesion molecules (VCAM1, ICAM1 and E-selectin) ${ }^{48}$, and further signals from endothelial cells and the endothelial glycocalyx (Box 3 ) that will attract and facilitate entry of more immune cells from the blood stream $^{2}$. Additionally, the extracellular matrix (ECM) that surrounds and supports cells in all tissues can be remodelled in response to RT. The cytokine TGF $\beta$ is important in this process, as well as being a key regulator of immunity in tissues, with levels modulated by RT (Box 4).

Overall, a certain level of inflammation appears to be necessary for promoting an antitumour immune response, the protection of RT-damaged tissues and initiation of normal tissue healing and remodelling. This state might be transient if followed by appropriate resolution of inflammation and injury of the normal tissues (Figure 1). However, the clinical situation is further complicated by protracted fractions of RT which can be extended over 6 or more weeks, which may be a factor leading to the establishment of chronic inflammation and hinder recovery of tissues and organs.

\section{Chronic immunological effects of RT on normal tissue}

After the RT-induced acute phase of inflammation within the TME and surrounding normal tissue, the immune response switches to attempt to resolve this acute phase, ideally without chronic inflammation and late organ toxicity. However, the reality of clinical practice is that late normal tissue toxicity is common, with severe chronic inflammation appearing 4-12 weeks after RT, which can lead to tissue fibrosis (4-12 months with progression over several years $)^{17,66}$. In the lung, this can lead to dyspnoea or even respiratory failure in $5-20 \%$ of patients treated with thoracic/chest $\mathrm{RT}^{7,67}$. In the gut, late onset mucosal enteritis associated 
with bleeding and chronic diarrhoea is reported in approximately $20-60 \%$ of patients, which can eventually lead to fibrosis or necrosis of parts of the intestine following pelvic RT ${ }^{68,69}$. Interestingly, RT-induced late side effects (e.g. bowel and lung injury leading to chronic diarrhoea or pneumonitis respectively) have been reported to occur local to but outside of the specific RT field up to several months post- $\mathrm{RT}^{9,11,19,70}$, potentially linked to immune effects spreading beyond the RT field.

The exact mechanisms for these delayed effects remain unknown, but the effects outside of the targeted RT field are potentially important when probing underlying mechanisms and suggest a systemic immune response may be important. Some evidence suggests that effects may be a consequence of the delayed cell senescence and mitotic catastrophe induced by $\mathrm{RT}^{7,71,72}$, promoting inflammatory responses and contributing to a failure of irradiated tissues to appropriately heal and resolve ongoing inflammation and injury ${ }^{2,67}$. Indeed, given the known induction of cellular senescence by $\mathrm{RT}^{73}$ and the importance of the immune system in clearance of senescent cells ${ }^{74}$, there are likely important mechanistic links between RTinduced cellular senescence, alterations in the immune system and chronic effects in tissues. However, the immunological consequences of this are less clear and require further investigation. The failed remodelling of tissues is potentially exacerbated with additional consecutive fractions of RT and sustained/propagated by TME-associated pro-fibrogenic cells and mediators $\mathrm{s}^{37,60,75}$.

The observed late effects of RT on normal tissue have long been known to depend not only on total RT dose but also on the dose per fraction, as well as the volume of tissue treated ${ }^{57,76}$. In addition, certain patients appear to be at higher risk of chronic inflammation and associated fibrosis after RT, potentially related to genetic mutations or polymorphisms (radiogenomics) in genes encoding for DNA repair mechanisms, ROS scavengers or immune mediators like TGF $\beta$, TNF, IL-18 or members of RAS signalling pathway ${ }^{67,71,77}$. The specific immunological aspects of chronic side effects in the lung and gut will be discussed in more detail below, given these organs are highly exposed during common thoracic, abdominal and pelvic RT treatments and the recent evidence of important immunological cross-talk between these mucosal sites ${ }^{78}$. 
$R T$-induced chronic immunopathology in the lung.

The irradiation of the surrounding lung tissue in the treatment of thoracic malignancies is common and can result in serious late normal tissue injury to the lung and heart. There are numerous reports investigating the underlying mechanisms of RT-induced lung toxicity leading to pneumonitis and fibrosis in preclinical models ${ }^{17,36,55}$. An important point to note is that the RT dose and timing may differ between the multitude of protocols used to study the immune effects of RT on the lung. However, some common themes in alteration of immune responses have emerged from different studies, which we summarise below.

The specialised lung alveolar epithelium is heavily infiltrated with immune cells, including specialised alveolar macrophages ${ }^{17}$. Of the two types of pneumocytes, type I make up the alveoli, while type II have precursor functions, which help tissue remodelling as well as producing a thin layer of protective surfactant that together with the more recently identified lung microbiota acts to maintain an efficient mucosal barrier ${ }^{79}$.

Following the initial RT-induced changes that may lead to an acute reduction in alveolar and immune cell numbers in the lung, at $\sim 4$ weeks, a substantial increase in the numbers of neutrophils, macrophages, monocytes and lymphocytes has been reported ${ }^{34,36,55,80}$. These immune infiltrations, often promoting type 2 immune responses, may persist for many weeks ( 32 weeks) at which point tissue remodelling, fibroblast proliferation and differentiation to myofibroblasts, deposition of collagen and fibrosis was apparent in susceptible mouse strains $^{28,81}$. Indeed, studies suggest that the balance between type 1 and type 2 immune responses might be critical for the development of fibrosis after $\mathrm{RT}^{32,54,80,81}$. Acute $\mathrm{RT}$ responses are associated with increased levels of Th1 cells and the type 1-associated cytokines IL-6, IL-12, TNF and IFN $\gamma^{32,43,71}$. Th1 cells and IFN $\gamma$ support an M1-like macrophage phenotype and together with Th17 cells are suggested to contribute to pneumonitis, persisting even for several weeks after $\mathrm{RT}^{32,43}$. However, fibrotic lung tissues, months after $\mathrm{RT}$ exposure, are often flooded with Treg cells, Th2 cells and the type 2-associated cytokines IL4 and IL-13 $32,43,81$. TGF $\beta, \mathrm{IL}-4$ and IL-13 support M2-like macrophage polarisation which produce IL-10 and more TGF $\beta$, the latter being a key fibrogenic mediator ${ }^{17,61,67}$ (Figure 2).

Several pro-inflammatory chemokines have also been shown to contribute to RT-induced chronic pneumonitis ${ }^{29,31}$. Enhanced expression of CXCR1 and CXCR2 was associated with neutrophil infiltration in mice. When both of these receptors (accounting for $\sim 60 \%$ of 
neutrophil infiltration) were pharmacologically antagonised, the pneumonitis following high dose (18 Gray, Gy) thoracic RT was reduced and survival was improved ${ }^{34}$. However, pulmonary fibrosis still progressed ${ }^{34}$, suggesting the likely involvement of additional immunological pathways and immune effector cells.

Inflammatory $\mathrm{CCR}^{+}$monocytes were shown to infiltrate irradiated lung in response to RTinduced IL-1 $\beta$-mediated CCL2 production. In mice lacking IL-1 $\beta$, its receptor IL-1R, or CCR2, post-irradiation-induced fibrosis was reduced ${ }^{55}$. In another study, CCL3 levels increased after RT and persisted up to 32 weeks which was associated with increased levels of IL-4 and IL-13 and polarisation of M2-like macrophages ${ }^{36}$. In mice lacking CCL3 or its receptor CCR1, a reduction in numbers of $\mathrm{CD}^{+} \mathrm{T}$ cells and macrophages was correlated with reduced proinflammatory cytokines, lower collagen deposition and better survival ${ }^{36}$. These effects appeared independent of $C D 4^{+} \mathrm{T}$ cell numbers, neutrophils, Treg and $\mathrm{B}$ cells and fibrosis was not seen in $\mathrm{Crr}^{-/}$mice ${ }^{36}$. Interestingly, when another CCL3 receptor, CCR5 was ablated, the mice developed an exacerbated fibrotic phenotype after single high dose of $\mathrm{RT}^{36}$, suggesting that the presence of CCR5 expression, usually found on $\mathrm{CD}^{+}$Th1 cells, monocytes and macrophages ${ }^{82}$, might provide some protection from fibrosis. Further, when IL-13 was directly targeted, mice were protected from fibrosis following fractionated irradiation ${ }^{83}$. In contrast, while the inhibition of IL-4 reduced M2-like macrophage levels, this did not reduce fibrosis after a single high dose ${ }^{28}$, implying a key role for IL-13 in driving RT-induced lung fibrosis. M2like macrophages are a major producer of TGF $\beta$, and levels of this pro-fibrotic cytokine were found to be elevated and persistent for at least 32 weeks in irradiated lungs ${ }^{29}$ but were reduced in fibrosis-free $\mathrm{Ccl}^{-/-}$or $\mathrm{Ccr}^{-/-}$mice $^{36}$.

More recently, alveolar and interstitial macrophages were found to contribute differentially to the development of RT-induced fibrosis ${ }^{80}$. In a radiation-induced lung fibrosis model in mice, depletion of alveolar macrophages via intranasal administration of clodronate liposomes had no effect on development of fibrosis, whereas depletion of interstitial macrophages via injection of an anti-CSF1R antibody significantly blocked RT-induced pulmonary fibrosis ${ }^{80}$. Together, studies described above support a key role for macrophages in the development of RT-induced pulmonary fibrosis and give insight into the phenotypical changes and specific subsets that are driving the disease. 
Whilst chronic inflammation has been shown to precede fibrotic events ${ }^{31}$, some proinflammatory mediators are suggested to be protective ${ }^{71,84}$. For example, M1-like macrophage-derived TNF produced during tissue healing has been linked to a reduction in expression of fibrosis-associated ECM genes, while Th1 cell-derived IFN $\gamma$ has been shown to downregulate TGF $\beta$ and suppress collagen synthesis ${ }^{71}$. However, it should be noted that these data were obtained using a bleomycin-induced lung fibrosis mouse model, which is characterised by accelerated fibrosis and potentially altered cytokine profile compared to RTinduced models ${ }^{84}$.

Thus, although the total RT dose, fractionation and delivery techniques vary considerably across studies, important general observations showing alterations in numerous innate and adaptive immune cells and the cytokines they produce have been observed in the lung postRT. The important consequences of these alterations to the immune system and their role in promoting interstitial RT-induced lung fibrosis requires further investigation.

\section{Immune mechanisms of RT-induced gut injury.}

Abdominal and pelvic RT is delivered to many cancer patients including patients with upper and lower gastrointestinal (GI) tumours, pancreatic cancers as well urinary and gynaecological malignancies. Some studies indicate that up to $90 \%$ of cancer survivors who have received pelvic RT may develop some symptoms suggestive of late RT-induced side effects ${ }^{68,69,85}$. While the acute symptoms are relatively easy to manage, chronic radiation-induced intestinal toxicity can be much more difficult to diagnose and treat ${ }^{66,68}$. Considering the extended time of development and progression of the RT-induced Gl effects (from 6 months to 3 years or even decades after RT exposure $)^{66,68,86}$ the pathophysiology appears complex, involving multiple cellular compartments (Figure 3).

The intestine is extremely susceptible to acute RT-induced injury, due at least in part to the high proliferation rate of intestinal epithelial cells lining the gut. The high turnover of these cells allows for recovery within weeks ${ }^{87,88}$. However, it is possible that the extent of the injury and repeated fractions of RT might prolong or interrupt the repair process ${ }^{86}$ and allow the leakage of the lumen content and the microbiota into the underlying intestinal tissue, which can induce inflammation to promote both acute and chronic damage $\mathrm{e}^{16,89}$. 
In a mouse model of intestinal injury, macrophage accumulation was observed starting from week 6 after fractionated pelvic RT, and persisted up to 30 weeks ${ }^{88}$. The macrophages were seen aggregating in close proximity to vessels and degenerated crypts, creating an inflammatory milieu and perhaps contributing to an abnormal division of crypts (crypt fission) and hindering tissue regeneration for weeks after RT. Similar observations were made within intestinal biopsies from cancer patients after RT and in other preclinical models ${ }^{16,88}$.

Increased expression of monocyte and macrophage-derived CXCL1 and CXCL2 chemokines that can attract neutrophils was observed in the fibrotic small bowel of patients with radiation enteritis ${ }^{16,90}$. CXCL8 (or its homologues in mice) can be produced by macrophages, but also by other immune cells, endothelial and epithelial cells. By binding to the CXCR1 and CXCR2 receptors, CXCL8 can promote recruitment of neutrophils, as seen in irradiated lungs $\mathrm{s}^{34}$. Following a high dose (27Gy) RT, in murine models of radiation-induced proctitis, CXCL1 and CXCL2 levels increased as early as $3 \mathrm{~h}$ post-RT exposure ${ }^{91}$. This was followed by increased numbers of neutrophils which were seen to persist within the irradiated tissue up to 26 weeks after RT and was also confirmed in biopsies from cancer patients receiving pelvic RT (45Gy in $\sim 2 \mathrm{~Gy}$ fractions $)^{91}$. However, as reported in the lung, increased neutrophil presence did not always correlate with detrimental effects and rather provided a degree of protection from excessive inflammation. In other models of inflammation, in mice lacking CXCR2, which show markedly impaired neutrophil infiltration, an exacerbated inflammatory response was observed, which correlated with reduced expression of cytokines associated with the resolution of inflammation and an increase in inflammatory macrophage accumulation ${ }^{92}$. Indeed, in a model of radiation-induced proctitis in mast cell deficient mice, increased neutrophil numbers were associated with reduced intestinal tissue damage ${ }^{91}$.

In mouse models of radiation-induced proctitis and in patients receiving RT, accumulation of mast cells was observed in the inflamed and fibrotic sections of the intestine ${ }^{91}$. However, in rat models, mast cells were severely depleted after fractionated RT, and mast cell-deficient animals developed severe acute mucosal damage but were protected from collagen accumulation following irradiation of the small intestine ${ }^{93}$. It is not certain if the differences in mast cell contribution to the intestinal RT-induced injury are due to the varied responses within the different sections of the gut, different RT regimes, species-specific or if there are additional factors involved. 
Intestinal eosinophils were seen to accumulate after single dose of RT and were reported to contribute to RT-induced intestinal fibrosis ${ }^{94}$. Eosinophils expressing CCR3 can be recruited primarily by endothelial cell-derived IL- 5 and CCL11, but also by IL-3 and GM-CSF and have been implicated in driving inflammation in inflammatory bowel disease (IBD) $)^{95}$. Recruitment of eosinophils into the inflamed gut appears independent of the microbiota and involves binding of eosinophil-expressed integrins to the intestinal endothelial adhesion molecule MADCAM-196. Secretion of cytolytic mediators from eosinophilic granules can mediate long term inflammation ${ }^{95}$. In mice lacking total eosinophils, CCR3 or the IL-5 receptor the RTinduced accumulation of eosinophils, intestinal wall thickening and collagen accumulation were reduced significantly 12 weeks post-RT ${ }^{94}$. Eosinophil-mediated intestinal injury was associated with RT-induced release of ATP from dying crypt cells, which in turn activated expression of CCL11 by pericryptal alpha-smooth muscle actin positive ( $\alpha$-SMA ${ }^{+}$) cells in the submucosa ${ }^{94}$, strengthening the role of the CCL11-CCR3 axis in eosinophil recruitment ${ }^{82}$. Activated $\alpha-\mathrm{SMA}^{+}$cells also expressed GM-CSF which stimulated eosinophils to express TGF $\beta$, accelerating the fibrogenic effects of $\alpha-S_{M A}$ myofibroblasts $^{94}$. Th2 cells and type 2 innate Iymphoid cells (ILC2s) are also a source of IL-5 in the intestine but the observed RT-induced eosinophilic recruitment was mediated in a Th2 cell-independent manner ${ }^{94}$. Even though the intestinal lamina propria harbours a pool of dedicated DCs and innate lymphoid cells (mainly ILC2) little is known about their role in RT-induced bowel toxicities.

The balance of Th1/Th2 cell responses and its correlation with chronic tissue injury ${ }^{32,81}$ was also evaluated in the gut after cumulative (4Gy $\times 3$ fraction/week) colorectal irradiation in rats $^{97}$. An early increase in CCL2, TNF and IL-1 $\beta$ was followed by an increase in neutrophil and macrophage numbers and was observed following RT, along with increased levels of the Th2associated chemokine CCR4 and the transcription factor GATA $3^{97}$. At this early stage, suppression of the Th1 cell-associated molecules IFNY, CXCR3, CXCL10 and T-bet was apparent ${ }^{97}$. This suppression of the Th1 cell-associated IFNY-STAT1 pathway and upregulation of Th2-type mediators persisted up to 26 weeks post-RT exposure and was correlated with detrimental changes in the intestine ${ }^{97}$. Additionally, there was also enhanced expression of suppressor of cytokine signalling 3 (SOCS3), which was associated with suppression of the IL10-STAT3 regulatory pathway ${ }^{97}$. This suppression appeared to tip the balance towards a Th2mediated immune response and chronic immune effects associated with fibrosis ${ }^{16,81}$. 
Similarly to irradiated lung, irradiated gut tissue is flooded with a myriad of proinflammatory cytokines, including IL-1 $\beta$, TNF and TGF $\beta^{29,30,32}$. An increase in the mRNA of TGF $\beta$ was reported transiently within epithelial and smooth muscle cells, but persisted in lamina propria, fibroblasts, and endothelial cells at least up to 26 weeks after RT in rats, and was associated with RT-induced dose-dependent fibrosis ${ }^{98}$. In rats and humans who developed late enteritis after abdominal fractionated RT, increased activation of matrix metalloproteinase 2 (MMP-2) and MMP-9 was associated with the high levels of TGF $\beta$ in the irradiated intestine and resulted in ECM and collagen deposition ${ }^{99}$. As stated in Box 4, certain $\alpha_{v}$ integrins are also key activators of TGF $\beta$. However, their role in TGF $\beta$-mediated RT-induced effects in the intestine is unclear at present.

RT and ROS-induced regions of hypoxia may also lead to tissue damage associated with an inflammatory microenvironment ${ }^{17,100}$. In a mouse model of radiation-induced enteritis, a selective deletion of hypoxia-inducible factor 1 alpha (HIF-1 $\alpha$ ) in endothelial cells, protected mice from RT-induced intestinal damage, and increased their survival ${ }^{101}$. This protection was associated with preserved vasculature, reduced hypoxia, reduced macrophage numbers and reduced levels of pro-inflammatory IL-1 $\alpha$, IL-1 $\beta$, IL-6 and TNF ${ }^{101}$.

Growing evidence suggests that shifts in the diversity of the intestinal microbiota following bowel irradiation may mediate local and even systemic immune effects $89,102,103$. Important immune effects of the microbiota can be mediated by various metabolites produced by the different types of microbes, of which the short-chain fatty acids (SCFAs) acetate, propionate and butyrate have been most studied ${ }^{104,105}$. Butyrate can promote recruitment and maturation of immune cells and was shown to enhance DC cross-priming efficiency after tumour irradiation and RT-induced anti-tumour $\mathrm{CD}^{+} \mathrm{T}$ cell responses, even in distant metastatic cancer sites outside of the RT field ${ }^{102}$.

The change in the diversity of microbiota and the breakdown of epithelial barriers can activate epithelial cell-microbial sensing capabilities and trigger expression of various receptors for microbial-associated patterns and a subsequent array of cytokines, chemokines and immune effectors ${ }^{106}$. In a model of faecal transfer, the transfer of microbiota from irradiated mice enhanced IL-1 $\beta$ and TNF expression in germ-free mice when compared with the transfer of microbiota from non-irradiated mice ${ }^{107}$. Administration of an IL-1R antagonist mitigated some of the RT-induced damage, supporting the role for IL-1 $\beta$ and microbiota 
perturbations as potential drivers of radiation-induced enteritis. In another study, total body irradiated mice were given faecal matter from non-RT-treated mice, which provided some protection from RT-induced GI side effects and extended survival ${ }^{108}$. All these data support the idea that the microbiome is key in regulating immune responses to RT, although the exact mechanisms and the mediators involved are still being uncovered.

Overall, the precise mechanisms leading to the chronic bowel inflammation months after the initial RT-induced bowel injury require further attention. Pathologically, some similarities are observed with other types of intestinal inflammation initiated by different causes such as Crohn's disease, ulcerative colitis or dietary enzymatic deficiencies. While RT-induced gut injury is used as a model of IBD, the extent of the overlap of the underlying mechanisms between these different conditions remains unclear ${ }^{15}$.

The potential for RT-induced immune cross-talk between lung and gut.

Recently, data has shown the potential for important immune-mediated cross-talk between distal organs with reports identifying cross-regulation of immunity between the gut and lung ${ }^{104,105,109-111}$. Indeed, RT-induced effects at distal organs, outside of the RT field have been described ${ }^{70,112}$. However, abscopal immune responses are rare. Thus, greater understanding of how RT can induce immune responses at distal sites may identify potential pathways that can be targeted to enhance abscopal responses.

The mechanisms of immunological cross-talk between organs are still poorly understood, but could involve the non-specific circulation of immune cells and immune mediators or more specific modulation of tissue-specific homing signals ${ }^{104,109}$. Examples of non-specific circulation might include the circulation of microbiota components and their products after RT-induced mucosal barrier damage, circulating cancer cells or progenitors and immature immune cells. Indeed, there is clear evidence that alterations in the gut microbiota can have significant effects on lung health and disease and vice-versa ${ }^{113}$. Thus, RT-induced changes to the microbiota are likely an important mechanism for gut-lung immunological cross-talk, and therefore a potential therapeutic target is manipulation of the microbiota post-RT to promote health. Additionally, certain circulating factors, for example heparinase or cytokines, can promote endothelial glycocalyx damage, facilitating immune cell infiltration in distal 
tissues ${ }^{24,65,114}$. Additionally, adhesion molecules and integrins that are ubiquitously expressed could also facilitate a non-tissue-specific cell recruitment.

More specific tissue-homing signals may also be important in RT-induced cross-talk between gut and lung. Activated antigen-bearing DCs migrate from tissues to local draining lymphoid structures. Within the gut, Peyer's patches and mesenteric lymph nodes are part of the gut-associated lymphoid tissues (GALT), which in conjunction with the bronchusassociated lymphoid tissue (BALT) is part of the mucosa-associated lymphoid tissue (MALT), which can facilitate connections between the intestine and lung ${ }^{115}$. Gut-derived DCs imprint T and B cells within the GALT by upregulation of their expression of integrin $\alpha_{4} \beta_{7}$ and CCR9. These bind to MADCAM1 and CCL25 respectively, which are mainly expressed in the small intestine $^{116}$ (Figure 4). Additionally, DCs that migrate from the lung to the BALT can imprint T cells to express CCR4, that can bind to CCL17 expressed by lung epithelial and endothelial cells ${ }^{117}$. The imprinting on B cells in the BALT was reported to result in upregulation of integrin $\alpha_{4} \beta_{1}$ and CCR10 that could bind to VCAM1 and CCL28 respectively, which are found within the colon but also lung tissue ${ }^{116}$. For B cells, additional expression of CD62L upon respiratory inflammation was linked to binding to the peripheral lymph node addresin (PNAd) that is abundant on endothelial cells within the BALT, homing these plasma cells back to the site of inflammation ${ }^{116}$.

Thus, DCs can imprint T and B cells to home back to local organs. However, there is also evidence that DC-mediated imprinting in the lung can promote migration of cells to the gut. Consequently, lung-derived DCs were able to imprint expression of the gut-homing integrin $\alpha_{4} \beta_{7}$ and CCR9 on co-cultured T cells in vitro and on adoptively transferred cells in vivo ${ }^{118}$. In a model of mouse respiratory influenza infection, intestinal immune injury was mediated by lung-derived CCR9+ $T$ cells that home to the intestine. Within the intestine, $T$ cells from the lung mediated IFNY-induced intestinal microbiota shifts that resulted in a Th17-mediated inflammatory response ${ }^{111}$.

These examples represent the plasticity of the tissue-specific homing signals and open up opportunities for 'cross-organ' targeting. This might be a potential mechanism also for the RT-induced immune effects observed at distal sites, outside of the irradiation field ${ }^{19,22}$. Likely many more homing signals take part in these targeted migration processes, but these remain to be elucidated. 


\section{The mitigation of RT-induced toxicity}

Despite normal tissue toxicity remaining a dose-limiting effect of RT and a major cause of morbidity for cancer survivors, current treatment options to alleviate these side effects are limited, with anti-inflammatory drugs commonly used. More recently, novel agents aimed at mitigating various underlying toxicities are in development ${ }^{8}$. These novel targets include the prevention of acute cell death (by targeting the p53 or NF-kB pathways), restoration of the redox equilibrium (for example, ROS scavengers and hypoxia pathway inhibitors), and modulation of inflammatory and fibrogenic signals (for example, TNF, TGF $\beta$ and collagen inhibition $)^{8}$.

TGF $\beta$ has been a longstanding target ${ }^{119}$ given that it is implicated as a mechanism of action $^{59}$ at multiple steps of RT-mediated effects; from regulating the balance between effector and suppressive immune responses to facilitating resolution of inflammation, healing of tissues, fibroblast proliferation and differentiation to myofibroblasts, ECM remodelling and fibrosis. However, there are potential toxicities related to the systemic and long-term inhibition of such pleiotropic and ubiquitously expressed molecules. Indeed, TGF $\beta$ dysregulation is implicated in several inflammatory conditions and mice lacking TGF $\beta 1$ die early in life from multi-organ inflammation ${ }^{120,121}$. Therefore, the targeting of TGF $\beta$ signalling has to be considered carefully and perhaps, locally administered specific inhibitors may be of benefit. For TGF $\beta$, there is great interest in targeting site and context-specific activators of this cytokine; for example, certain integrins that can liberate the active TGF $\beta$ cytokine from its latent complex ${ }^{121}$. In support of this approach, mice lacking expression of integrin $\alpha_{v} \beta_{6}$ are completely protected from RT-induced lung fibrosis ${ }^{122}$.

Some other promising immune targets may include specific cell subset depletion at the right phase of the immune response by targeting chemokines or their receptors. Examples include inhibition of $\mathrm{CCR} 2$ and $\mathrm{CCR} 5^{33}$, which in preclinical models led to reduced infiltrating monocytes and fewer M2-like tumour-associated macrophages within the TME, which could therefore target both inflammatory and pro-fibrotic immune cells. Considering the apparent redundancy and specificity of the chemokine families ${ }^{82,123}$ potentially the best therapeutic strategies would involve targeting several of the collaborative chemokines in order to inhibit the recruitment of a specific immune cell subset. 
Every strategy to modulate normal tissue toxicity should aim to maintain the early efficient anti-tumour responses while mitigating the late excessively inflammatory effects. This temporal separation may allow for a window of opportunity to enhance the RT therapeutic index and prevent the limiting adverse effects.

\section{Future directions}

The recent "breakthrough status" of immune check point inhibitors and other emerging immuno-oncology (IO) agents have provided an unprecedented opportunity to combine these novel agents with RT to further enhance the anti-tumour immune response. Indeed, there are now thousands of clinical trials ongoing or in development combining RT and IO agents. Most of these studies have pragmatic designs and fail to address the important questions of RT dose, fractionation, optimal scheduling of the 10 agent, and few have translational research to address fundamental mechanisms of immunological response and resistance ${ }^{4}$. Furthermore, little attention has been given to the potential for 10 agents to increase immune-mediated RT-induced toxicities. The majority of published RT and IO agents trials have not observed substantially increased normal tissue toxicity. However, the importance of dose-limiting normal tissue toxicity was recently illustrated in the Phase I PLUMMB trial (Pembrolizumab in Muscle-invasive/Metastatic Bladder cancer) (NCT02560636), where patients have been treated with a combination of weekly 6Gy per fraction RT and the anti-PD-1 mAb pembrolizumab in patients with metastatic bladder cancer. The trial was paused after only 5 patients were treated, as significant toxicity was observed (urinary toxicity attributable to therapy in 2 patients, rectal perforation in another) ${ }^{124}$. This study illustrates the need to carefully investigate the effects of RT total dose, dose per fractions and RT treatment volumes when combining with IO agents, especially when normal tissues such as bladder, bowel and lung are irradiated.

In order to make rapid progress and minimise such severe normal tissue toxicity in the clinic, preclinical RT delivery platforms using in vivo orthotopic tumour models will likely prove very useful. As mentioned (Box 1), the dose and fractionation of RT is crucial in both tumour outcome and normal tissue toxicity. Such animal models will allow us to test how RT total dose and dose per fraction, in combination with 10 agents, affect anti-tumour efficacy and normal tissue toxicity, and inform clinical trial design to reduce the risk to patients. This is perhaps most important, when testing higher dose per fraction used in stereotactic ablative 
radiotherapy $(S A B R)^{125}$. Animal models such as fibrosis-susceptible mouse strains and other genetic backgrounds ${ }^{28,81}$ may also play a role in informing clinical trial design about RT dose per fraction and scheduling questions. Partial tumour irradiation or varied doses may also be important emerging approaches that require more thorough investigation ${ }^{126}$. The hypothesis that requires further testing is that partial tumour irradiation or differential higher and lower doses of RT to the same tumour may lead to preferential anti-tumour immune responses and limit normal tissue toxicity (so-called RADSCOPAL effect).

The clinical use of proton beam therapy (PBT) is increasing, but the immunological and normal tissue toxicities of PBT remain unexplored and require urgent further investigation. FLASH radiotherapy is gaining increasing interest and involves the ultra-fast delivery at dose rates several orders of magnitude higher than those currently used in clinical practice ${ }^{127}$. Emerging data suggest that ultra-fast dose rates allow normal tissue tolerance levels to be exceeded with a greater probability of tumour control and little or no normal tissue damage. The underlying mechanisms of this normal tissue sparing and the effects of FLASH on the surrounding immune contexture are unknown and require further investigation.

There is currently little research focused on addressing the potentially important local and systemic "organ to organ" communication that RT may induce. This is novel but potentially important area of research, given the increasing use of 10 agents, and research is needed to further understand the immunological mechanisms that might underlie both acute and late toxicity which are often the dose-limiting toxicity in organs such as bowel and lung. The advances in deep phenotyping, allowing more in-depth analysis of the complex immune network and heterogeneity in tumours ${ }^{128}$ and how that might be influenced by RT, is likely to result in new insights regarding the underlying mechanisms.

In conclusion, these are exciting times with new opportunities to deliver RT in an entirely different way from much of last century, where the focus has been on enhancing the ability of RT to directly kill cancer cells. The understanding of RT-induced immune responses in the TME and surrounding normal tissues is likely to be critical to further enhancing the efficacy of RT. Key to success will be manipulating the immune system to increase the anti-tumour immune responses and decrease or modulate the RT-induced inflammatory responses in normal tissues. To achieve this goal, an enhanced understanding of the mechanisms 
underlying anti-tumour immune responses induced by RT and normal tissue organ toxicities will help identify targets for therapeutic interventions. Novel immune-directed therapeutics that are able to enhance the anti-tumour immune response and ameliorate the RT-induced immune-related acute and late toxicity would greatly enhance the therapeutic ratio of RT. Such therapeutic manipulation of the immune response to RT holds a great promise in transforming modern cancer care by improving cure rates and enhancing the quality of life of cancer survivors. 
Figures

ACUTE PHASE
tumour and early normal tissue responses $\begin{gathered}\text { CHRONIC PHASE } \\ \text { late normal tissue responses }\end{gathered}$

Tissue damage $>$ Inflammation $>$ Repair $>$ Recurrent inflammation
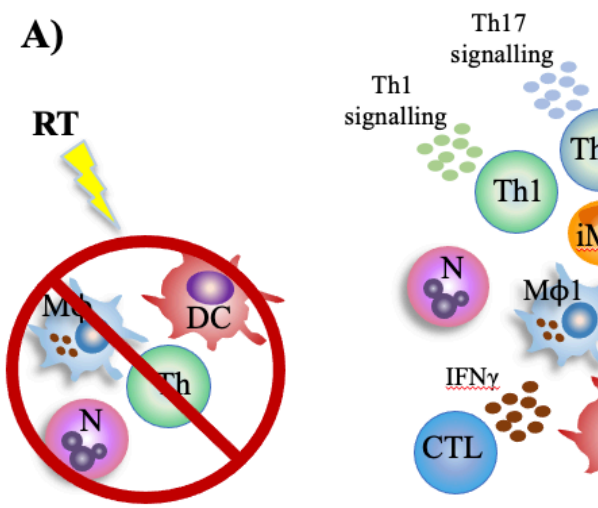

Th17

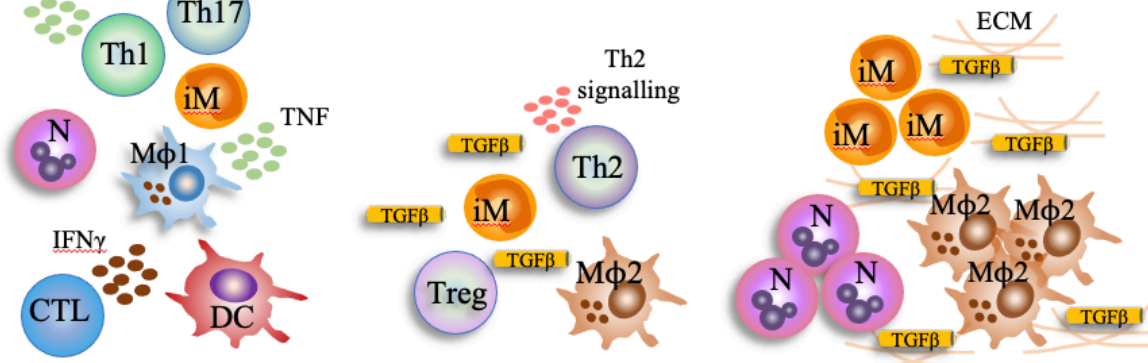

Immune cell depletion $\rightarrow$ immune cell recruitment/proliferation $\rightarrow$ tissue repair $\rightarrow$ recurrent chronic inflammation

B)

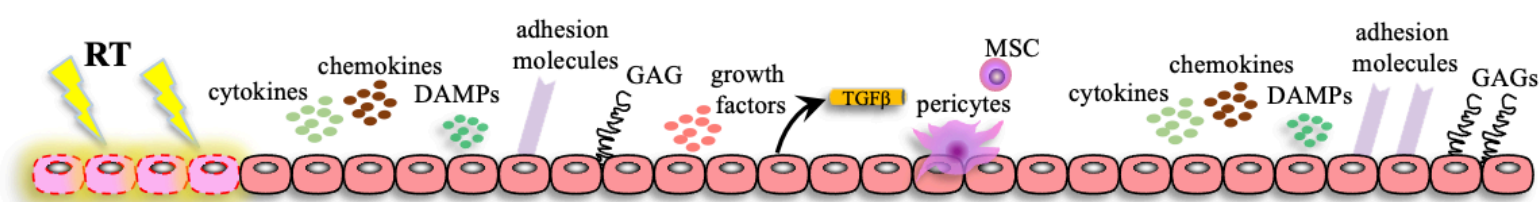
Endothelial damage $\rightarrow$ endothelial signalling and immune infiltration $\rightarrow$ endothelial repair $? \rightarrow$ secondary damage

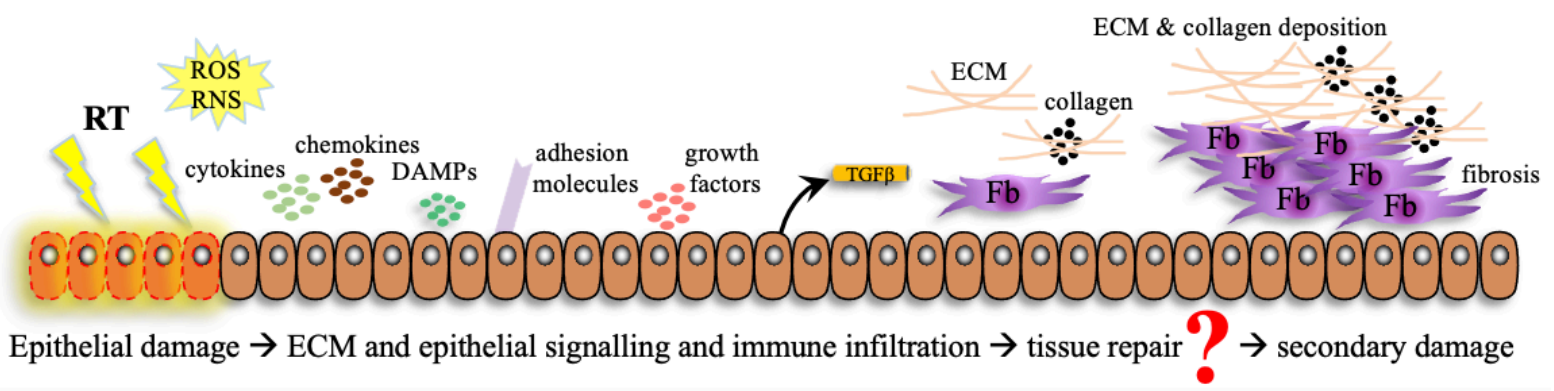

Figure 1. Timeline of acute and late radiotherapy (RT)-induced effects encompassing several

tissue components. A) RT has an initial cytotoxic effect on resident immune cells and leads to transient cell death. The initial pro-inflammatory responses are more of Th1/Th17 phenotype following the recruitment of immune cells into the tissue facilitated by chemokines, glycocalyx and adhesion molecules. In the second phase, the immune responses switch to Th2 for resolution of inflammation and initiation of tissue healing. This stage is facilitated by activation and release of TGF $\beta$ by multiple cells and from extracellular matrix (ECM) stores 
(Box 4). B) Soon after RT exposure, the endothelial and epithelial damage leads to the release of damage-associated molecular patterns (DAMPs), immune mediators (cytokines/chemokines) and upregulation of adhesion molecules that will activate and attract immune cells into the tissue. In time, the RT-induced damage of these barriers facilitates chronic immune cell recruitment (Box 2,3). Moreover, RT-induced wounds do not heal well and often a chronic inflammation persists or recurs in waves and contributes to secondary damage and fibrosis. This stage can perpetuate for months/years and the full mechanism leading to the late toxicities remains unknown. ROS - reactive oxygen species, RNS - reactive nitrogen species, GAG - glycosaminoglycan, MSC - mesenchymal stem cell, Fb - fibroblast, iM - inflammatory monocyte, CTL - cytotoxic T cell, DC - dendritic cell, N - neutrophil, MФ1, MФ2 - macrophage type 1 and 2, Th - T helper cell. 


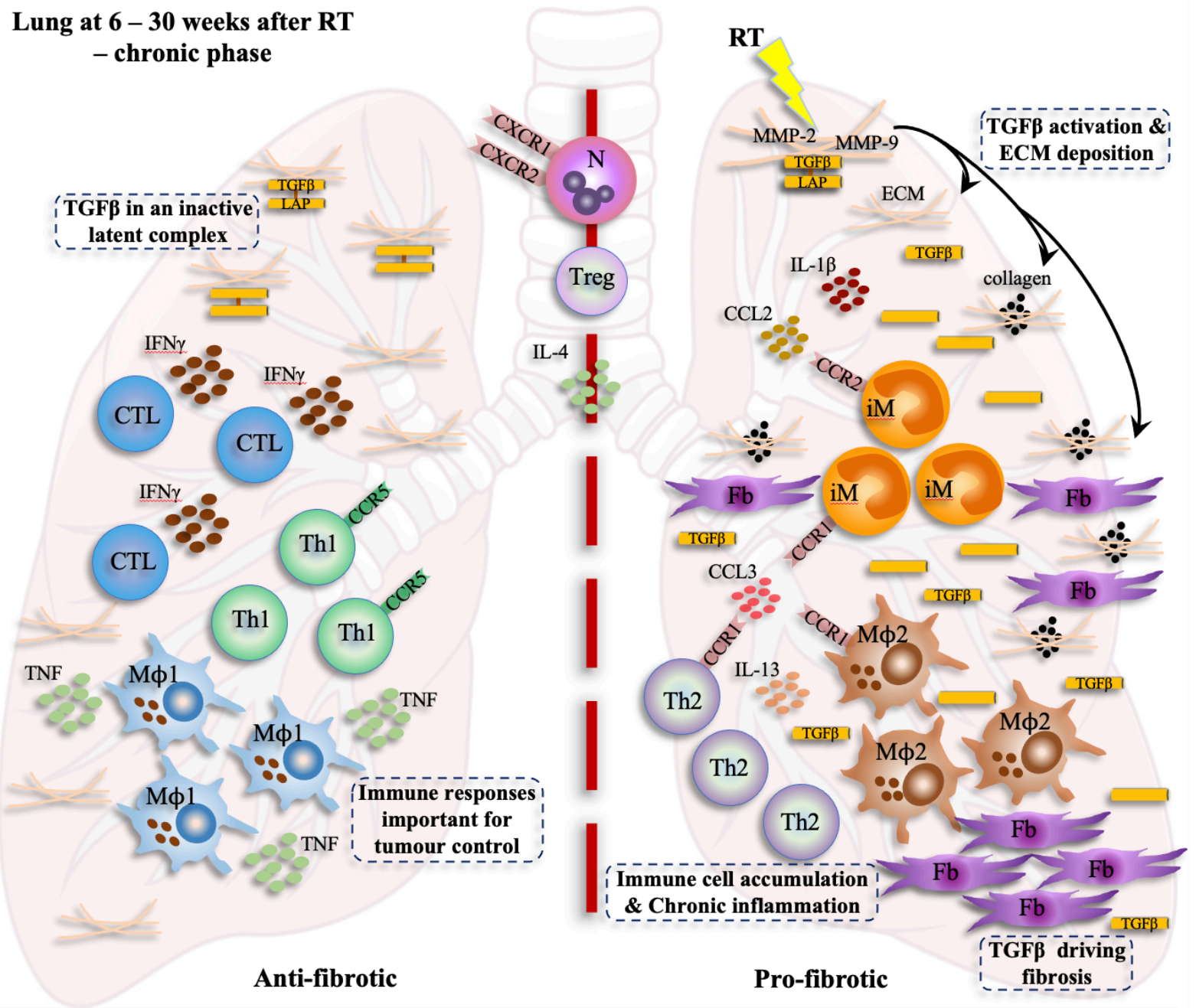

Figure 2. Immune cells and mediators involved in radiotherapy (RT)-induced chronic inflammation and fibrosis of the lung. Initially, RT can activate the extracellular matrix (ECM) metalloproteinases (MMP) and integrins that liberate TGF $\beta$ from the latency associated peptide (TGF $\beta$-LAP) complex tethered to ECM proteins. The active form of TGF $\beta$ can promote differentiation of fibroblasts $(\mathrm{Fb})$ to myofibroblasts leading to collagen deposition, ECM remodelling and eventually fibrosis. Inflammatory monocytes (iM) and type 2 macrophages

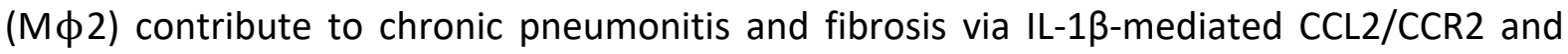
CCL3/CCR1 axis. Abundance of IL-13 and TGF $\beta$ (but not IL-4) is supportive of $M \phi 2$ differentiation and contributes further to pro-fibrotic events. High numbers of neutrophils $(N)$ are seen during acute and chronic pneumonitis, however in mice lacking neutrophils, the fibrosis still occurs, suggesting only partial, if any, involvement of neutrophils in the process. Similarly, the presence of regulatory T cells (Treg) is not directly associated with fibrosis. However, the accumulation of Th2 cells expressing CCR1 is implicated in driving the fibrotic 
processes. In contrast, the presence of Th1 $\mathrm{CD}^{+} \mathrm{T}$ cells expressing CCR5, M $\phi 1$, cytotoxic $\mathrm{T}$ cells (CTL), and their associated mediators TNF and IFN $Y$ are associated with some protection from the chronic fibrosis. 


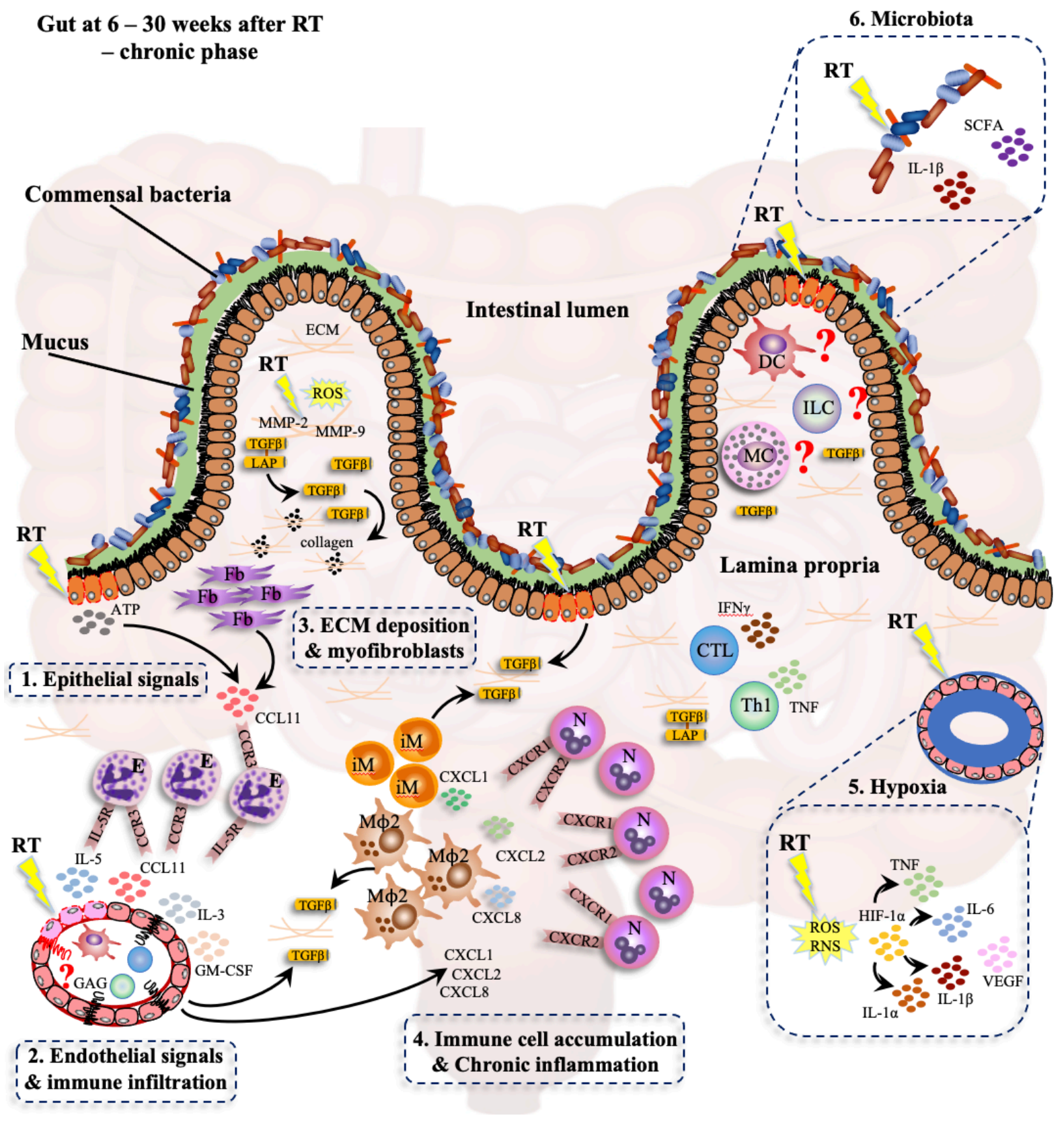

Figure 3. Immune aspects involved in radiotherapy (RT)-induced chronic inflammation and intestinal damage. $(\mathbf{1}, \mathbf{2})$ RT-induced damage of endothelial and epithelial cells results in the release of immune mediators that activate and recruit immune cells. (3) RT releases ROS/RNS from distressed cells leading to activation of matrix metalloproteinases (MMP) that liberate TGF $\beta$ from its inactive LAP-complex. TGF $\beta$ acts on fibroblast $(\mathrm{Fb})$ proliferation, and promotes their differentiation to myofibroblasts, resulting in collagen and extracellular matrix (ECM) deposition and eventually fibrosis. (4) Eosinophils (E) bearing IL-5 receptor and CCR3 bind to IL-5 and CCL11 enhanced after RT. Chronic accumulation of inflammatory monocytes (iM) and differentiation of type 2 macrophages (M\$2) happens upon RT-induced release of TGF $\beta$ from 
multiple sources. Endothelial cells, iM and $\mathrm{M} \phi 2$ produce chemokines that attract neutrophils (N). (5) RT and ROS/RNS accumulation contribute to hypoxia and activation of hypoxiainducible factor-1 alpha (HIF-1 $\alpha$ ) that can result in upregulation of several immune mediators and vascular endothelial growth factor (VEGF) contributing to angiogenesis and immune cell recruitment. Involvement of dendritic cell (DC), mast cell (MC) and innate lymphoid cell (ILC) is poorly understood. The presence of Th1 and cytotoxic T cell (CTL) does not have deleterious effects. (6) RT induces microbiota dysbiosis leading to the release of short-chain fatty acid (SCFA) and IL-1 $\beta$, propagating immune responses. 


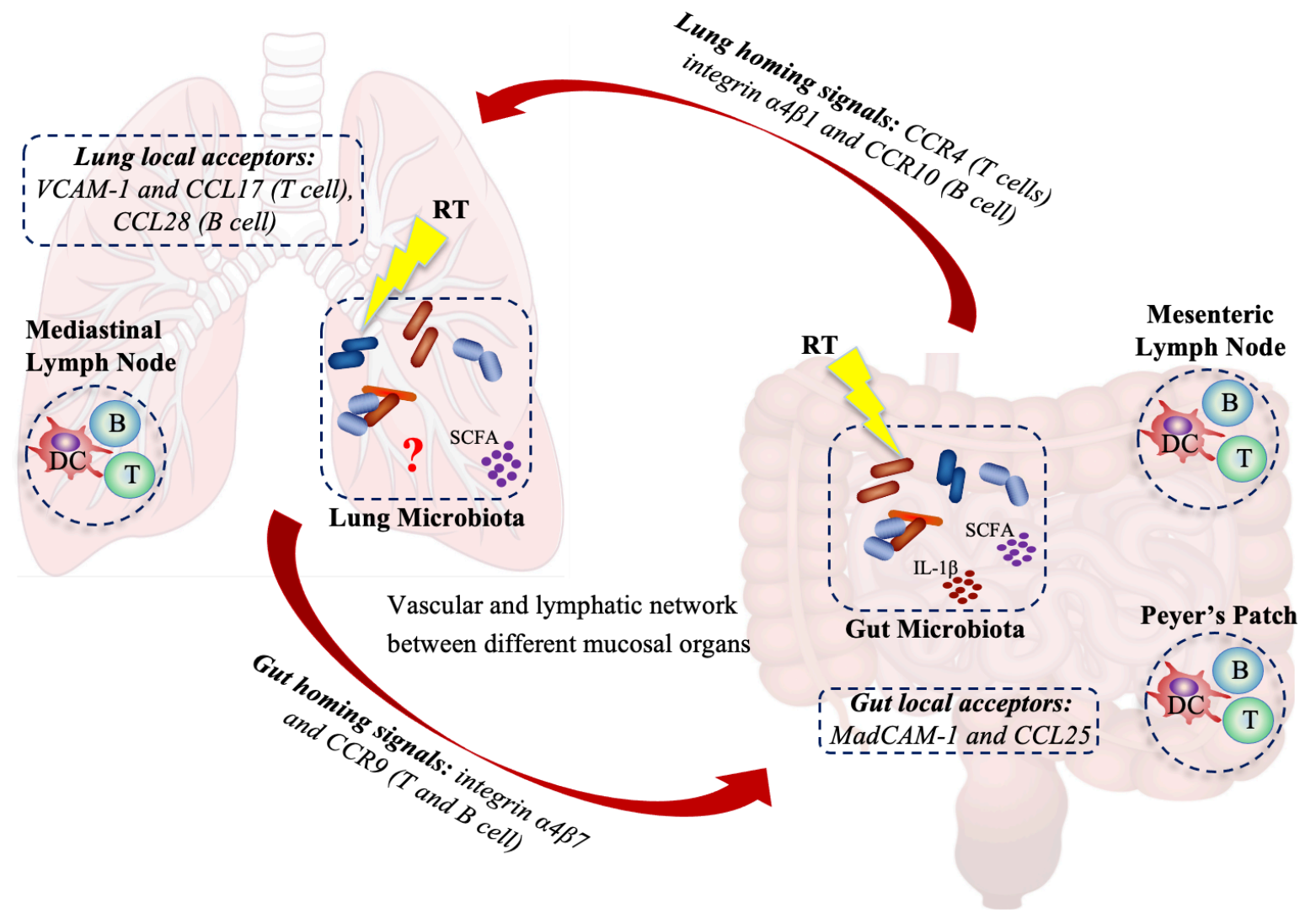

Figure 4. Potential homing signals for immune cross-talk between the mucosal sites of lung and gut. Few homing signals imprinted in the local draining lymph nodes by dendritic cells (DC) onto $\mathrm{T}$ and $\mathrm{B}$ lymphocytes are known for homing to the tissue of origin. Mucosal sites are hypothesised to form a network with potential migratory interactions. Reports show a possibility of cross-imprinting of the known homing signals to divert the traffic of immune cells across organs. This might be a potential mechanism also for the RT-induced immune responses observed at distal sites, outside of the irradiation field. Additionally, RT has been shown to influence local and distal diversity of microbiota populations. These shifts might be due to the circulating products of microbiota like short-chain fatty acid (SCFA) or IL-1 $\beta$ traveling within the mucosal system between the gut and the lung. At this stage, the full mechanism of these homing signals and the gut-lung cross-talk remains unknown. 


\section{Boxes}

\section{Box 1. Radiotherapy dosing regimes and preclinical models}

Current clinical RT plans include fractionated doses, often 1.8-2Gray (Gy) fractions delivered over a course of several weeks. However, these lower dose hyperfractionated regimes might not induce sufficient immune control in more resistant types of cancers and hypofractionated higher doses (3-20Gy/fraction) are used in fewer increments.

More recently, image-guided radiotherapy (IGRT), intensity-modulated radiotherapy (IMRT), stereotactic ablative radiotherapy (SABR), FLASH RT and proton beam therapy (PBT), enable higher intensity, more precise and focused radiation to be delivered to a smaller target volume in fewer doses ${ }^{14}$. These approaches are aimed at better protecting healthy tissues, with data from preclinical models and clinical settings suggesting improved tumour control $^{127,129}$. However, such technology is still in the early stages and better understanding of the biological responses, especially how such methods alter the immune system, is required to identify potential therapeutic targets that could be modulated in synergy with these RT delivery methods. To this end, complementary preclinical models will be essential.

Advances in preclinical models have provided important insights into the biological effects of RT that might be translatable to human clinical benefit. However, different dose and fractionation protocols are frequently used across preclinical studies, leading to contrasting results. For example, many preclinical models using low doses of RT ( $<2 \mathrm{~Gy})$ show overall immunostimulating effects, can reprogram tumour-associated macrophages from an M2-like to M1-like phenotype, and have the ability to induce tumour control ${ }^{42,126,130}$. However, some studies using higher RT doses (>8Gy), although also showing tumour control, can result in a more immunosuppressive TME, greater M2-like macrophage polarisation and greater normal tissue toxicity (e.g. late lung and gut inflammation and fibrosis) ${ }^{17,57,66,130,131}$. Furthermore, many animal studies use RT doses of $>10 G y$ to induce sufficient acute effects that result in chronic inflammation as a means to study mechanisms of RT-induced fibrotic changes ${ }^{36,55,80,91,94}$. Thus, care needs to be taken when comparing different preclinical studies, based on precise methodology used.

More recently, matching the advances of clinical RT delivery, preclinical models are starting to use fractionated RT doses (e.g. $3 \times 8 \mathrm{~Gy})^{97,99}$, and in combination with potential 
immunomodulating agents ${ }^{4,76}$. Additionally, small animal radiation research platform (SARRP) allows delivery of image-guided precise X-rays, more closely matching clinical delivery patterns. The use of proton irradiators to deliver higher energy RT and more efficient stimulation of the immune responses ${ }^{14,132}$ is likely to become more common in preclinical models. Thus, future work using preclinical models of RT is likely to mirror the technological advances seen in the clinic, and will uncover important mechanisms by which new RT technology promote beneficial responses.

\section{Box 2. RT-induced vascular and epithelial changes}

RT-related effects on cells of the vasculature and epithelial barriers are often important contributors to the propagation of inflammatory changes and driving immune cell recruitment.

Cells associated with the vasculature, like bone marrow-derived endothelial progenitors, mesenchymal stem cells (MSCs) and vascular smooth muscle precursors (pericytes), can be attracted to the site of injury to aid the initiation of tissue remodelling after $\mathrm{RT}^{2}$. Pericytes function to stabilise and regulate vascular permeability and endothelial proliferation in response to injury and can be attracted to the site by platelet-derived growth factor $\beta$ (PDGF $\beta), T$ TGF $\beta$, and CXCL12 released by activated endothelial cells and platelets ${ }^{133,134}$. Pericytes also contribute to collagen production and together with the RT-induced endothelial activation can lead to endothelial-to-mesenchymal transition (EndoMT), the first stage of normal vascular wound healing. This may lead to tissue fibrosis via excessive differentiation of fibroblast to activated myofibroblasts expressing $\alpha$-smooth muscle actin ( $\alpha$ SMA) and an excessive collagen deposition within $\mathrm{ECM}^{2,31,67}$. Tissue resident fibroblasts and myofibroblasts are closely associated with and are probably the biggest producers of ECM components.

Epithelial cells can respond to RT insult in a similar way as endothelial cells, resulting in a breakdown of tissue barriers. The disruption of epithelial barriers after RT leads to an additional influx of immune cells into tissues, but can cause the microbiota to enter tissues at mucosal barrier sites ${ }^{79,89,108,135}$. This in turn can lead to resident microbiota dysbiosis and introduce a potential infectious threat that evokes an immune response. RT can also stimulate 
epithelial-to-mesenchymal transition (EMT), that is activated by TGF $\beta$ signalling and leads to collagen and ECM deposition and eventually to fibrosis ${ }^{55,61,67}$. All these events are known to be RT dose-dependent, with widely different effects observed with lower or higher RT doses $^{57,76}$.

\section{Box 3. The glycocalyx}

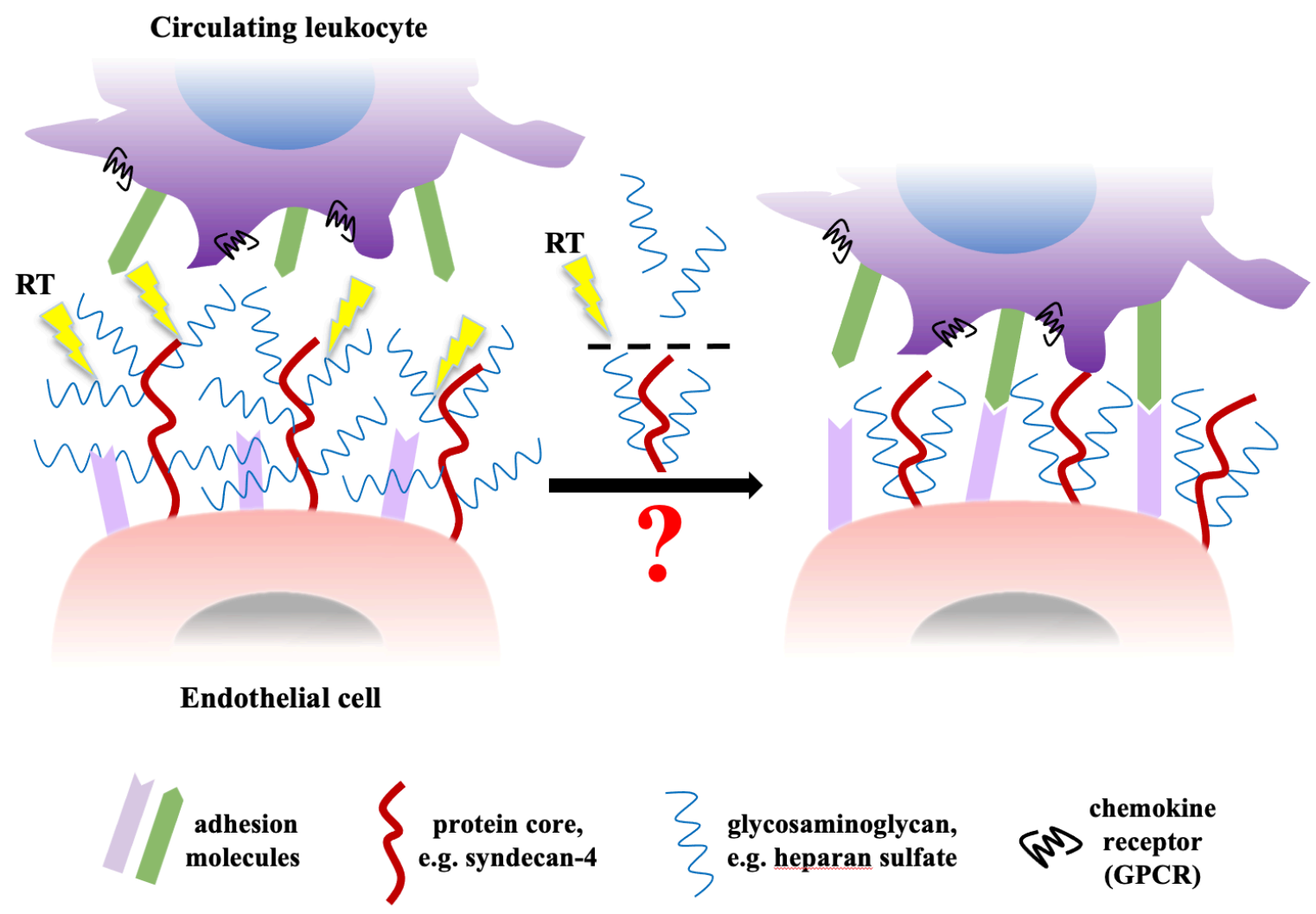

The glycocalyx is an extracellular matrix (ECM) structure that controls vascular permeability and chemokine presentation ${ }^{24}$. The glycocalyx barrier lines blood vessels and in a resting state inhibits leukocyte adhesion to the endothelium, thus preventing leukocyte recruitment to underlying tissues. The glycocalyx is composed of proteoglycans, protein cores embedded in the endothelial membrane, for example syndecans, decorated with glycosaminoglycan (GAG) sugar chains. GAGs are un-branched linear chains of repeating disaccharide units that are modified with differential sulphation patterns ${ }^{136}$ and are important in binding to and presenting chemokines ${ }^{114}$.

During inflammation GAG chains within the glycocalyx are structurally modified, via changes in their sulphation patterns, to facilitate specific chemokine presentation and 
leukocyte recruitment ${ }^{123}$. RT has been shown to reduce GAG levels and produce alteration in GAG sulphation ${ }^{137}$. Also, RT-induced production of ROS can degrade the glycocalyx and facilitate leukocyte recruitment to underlying tissues ${ }^{24,71}$.

The glycocalyx and its content are likely altered in response to RT and may be key to both the beneficial and negative effects of RT via regulation of immune cell recruitment.

\section{Box 4. ECM remodelling and immune regulation by TGF $\beta$}

Extracellular matrix (ECM) surrounds and supports cells of all tissues and organs ${ }^{138}$. Endothelial cells of vasculature and lymphatics and epithelial cells lining organs are tightly connected with the ECM. Anchored to the intracellular actin cytoskeleton, via transmembrane integrin receptors, are adhesive glycoproteins (like fibronectin, laminin) that connect to structural collagen fibres and proteoglycans ${ }^{138}$. There are also associated proteolytic enzymes (like matrix metalloproteinases, MMPs) that upon activation may result in abnormal ECM remodelling. A central cytokine in remodelling of the ECM, but also in regulating the immune system, is TGF $\beta^{59}$. This cytokine is produced by many cells (e.g. fibroblasts, pericytes, epithelial and immune cells) but is always secreted in an inactive form, with the latency associated protein (LAP) portion of TGF $\beta$ wrapping around the active cytokine to block the ability of TGF $\beta$ to bind to its receptor ${ }^{139}$. This latent cytokine complex is often tethered to ECM components from which it can be cleaved and activated by various integrins and $\mathrm{MMPs}^{59,140}$ and both have been shown to be upregulated after $\mathrm{RT}^{37,58,99,122}$.

Immune cells including Treg cells, DCs, monocytes and macrophages are known to express the integrin $\alpha_{v} \beta_{8}$ which has the ability to activate TGF $\beta^{139}$. Additionally, expression of integrin $\alpha_{v} \beta_{6}$ on intestinal and lung epithelial cells also contributes to TGF $\beta$ activation. TGF $\beta$ is crucial for maintaining tolerogenic immune responses and facilitates resolution of inflammation and wound healing, relevant for early and intermediate responses after RT exposure of normal tissues $^{59,61}$. Dysregulated TGF $\beta$ activation has been reported in several infection and inflammatory states including inflammatory bowel disease (IBD) and idiopathic pulmonary fibrosis $^{120,139}$. Similar dysregulation of TGF $\beta$ might occur in irradiated tissues, where increased levels of TGF $\beta$ have been reported ${ }^{98,141}$. Thus, accumulation of bioactive TGF $\beta$ is thought to 
be central in the processes leading to the late toxicities of irradiated normal tissues often associated with recurrent inflammation and fibrosis. 


\section{References}

1. Galluzzi, L., Buqué, A., Kepp, O., Zitvogel, L. \& Kroemer, G. Immunogenic cell death in cancer and infectious disease. Nat. Rev. Immunol. 17, 97-111 (2017).

2. Guipaud, O. et al. The importance of the vascular endothelial barrier in the immuneinflammatory response induced by radiotherapy. Br. J. Radiol. 20170762 (2018) doi:10.1259/bjr.20170762.

3. Reits, E. A. et al. Radiation modulates the peptide repertoire, enhances MHC class I expression, and induces successful antitumor immunotherapy. J. Exp. Med. 203, 1259-1271 (2006).

4. Romano, E., Honeychurch, J. \& Illidge, T. M. Radiotherapy-Immunotherapy Combination: How Will We Bridge the Gap Between Pre-Clinical Promise and Effective Clinical Delivery? Cancers 13, 457 (2021).

5. Rodríguez-Ruiz, M. E., Vanpouille-Box, C., Melero, I., Formenti, S. C. \& Demaria, S. Immunological Mechanisms Responsible for Radiation-Induced Abscopal Effect. Trends Immunol. 39, 644-655 (2018).

6. $\quad$ Reynders, K., Illidge, T., Siva, S., Chang, J. Y. \& De Ruysscher, D. The abscopal effect of local radiotherapy: using immunotherapy to make a rare event clinically relevant. Cancer Treat. Rev. 41, 503-510 (2015).

7. De Ruysscher, D. et al. Radiotherapy toxicity. Nat. Rev. Dis. Primer 5, 13 (2019).

8. Montay-Gruel, P., Meziani, L., Yakkala, C. \& Vozenin, M.-C. Expanding the therapeutic index of radiation therapy by normal tissue protection. Br. J. Radiol. 20180008 (2018) doi:10.1259/bjr.20180008.

9. Najafi, M. et al. Mechanisms of inflammatory responses to radiation and normal tissues toxicity: clinical implications. Int. J. Radiat. Biol. 94, 335-356 (2018).

10. Sharma, P. \& Allison, J. P. The future of immune checkpoint therapy. Science 348, 56-61 (2015).

11. Carvalho, H. \& Villar, R. Radiotherapy and immune response: the systemic effects of a local treatment. Clinics 73, (2018).

12. Dovedi, S. J. et al. Fractionated Radiation Therapy Stimulates Antitumor Immunity Mediated by Both Resident and Infiltrating Polyclonal T-cell Populations when Combined with PD-1 Blockade. Clin. Cancer Res. 23, 5514-5526 (2017).

13. Formenti, S. C. \& Demaria, S. Systemic effects of local radiotherapy. Lancet Oncol. 10, 718726 (2009).

14. Tsoutsou, P., Montay-Gruel, P. \& Vozenin, M.-C. The Era of Modern Radiation Therapy: Innovations to Spare Normal Tissues. in Radiation Oncology (ed. Wenz, F.) 1-15 (Springer International Publishing, 2019). doi:10.1007/978-3-319-52619-5_70-1.

15. Ferreira, M. R., Muls, A., Dearnaley, D. P. \& Andreyev, H. J. N. Microbiota and radiationinduced bowel toxicity: lessons from inflammatory bowel disease for the radiation oncologist. Lancet Oncol. 15, e139-e147 (2014).

16. François, A., Milliat, F., Guipaud, O. \& Benderitter, M. Inflammation and Immunity in Radiation Damage to the Gut Mucosa. BioMed Res. Int. 2013, 1-9 (2013).

17. Giuranno, L., Ient, J., De Ruysscher, D. \& Vooijs, M. A. Radiation-Induced Lung Injury (RILI). Front. Oncol. 9, 877 (2019).

18. Coates, P. J., Rundle, J. K., Lorimore, S. A. \& Wright, E. G. Indirect Macrophage Responses to Ionizing Radiation: Implications for Genotype-Dependent Bystander Signaling. Cancer Res. 68, 450-456 (2008).

19. Nikitaki, Z. et al. Systemic mechanisms and effects of ionizing radiation: A new 'old' paradigm of how the bystanders and distant can become the players. Semin. Cancer Biol. 3738, 77-95 (2016).

20. Gong, T., Liu, L., Jiang, W. \& Zhou, R. DAMP-sensing receptors in sterile inflammation and inflammatory diseases. Nat. Rev. Immunol. 20, 95-112 (2020). 
21. Cao, X. Self-regulation and cross-regulation of pattern-recognition receptor signalling in health and disease. Nat. Rev. Immunol. 16, 35-50 (2016).

22. Deng, L. et al. STING-Dependent Cytosolic DNA Sensing Promotes Radiation-Induced Type I Interferon-Dependent Antitumor Immunity in Immunogenic Tumors. Immunity 41, 843852 (2014).

23. Böttcher, J. P. et al. NK Cells Stimulate Recruitment of cDC1 into the Tumor Microenvironment Promoting Cancer Immune Control. Cell 172, 1022-1037.e14 (2018).

24. Marki, A., Esko, J. D., Pries, A. R. \& Ley, K. Role of the endothelial surface layer in neutrophil recruitment. J. Leukoc. Biol. 98, 503-515 (2015).

25. Wirsdörfer, F., de Leve, S. \& Jendrossek, V. Combining Radiotherapy and Immunotherapy in Lung Cancer: Can We Expect Limitations Due to Altered Normal Tissue Toxicity? Int. J. Mol. Sci. 20, 24 (2019).

26. Krysko, D. V. et al. Immunogenic cell death and DAMPs in cancer therapy. Nat. Rev. Cancer 12, 860-875 (2012).

27. Chiang, C.-S. et al. Irradiation Promotes an M2 Macrophage Phenotype in Tumor Hypoxia. Front. Oncol. 2, (2012).

28. Groves, A. M., Johnston, C. J., Misra, R. S., Williams, J. P. \& Finkelstein, J. N. Effects of IL-4 on pulmonary fibrosis and the accumulation and phenotype of macrophage subpopulations following thoracic irradiation. Int. J. Radiat. Biol. 92, 754-765 (2016).

29. Lierova, A. et al. Cytokines and radiation-induced pulmonary injuries. J. Radiat. Res. (Tokyo) (2018) doi:10.1093/jrr/rry067.

30. Ong, Z. et al. Pro-inflammatory cytokines play a key role in the development of radiotherapyinduced gastrointestinal mucositis. Radiat. Oncol. 5, 22 (2010).

31. Rubin, P., Johnston, C. J., Williams, J. P., McDonald, S. \& Finkelstein, J. N. A perpetual cascade of cytokines postirradiation leads to pulmonary fibrosis. Int. J. Radiat. Oncol. 33, 99-109 (1995).

32. Schaue, D., Kachikwu, E. L. \& McBride, W. H. Cytokines in Radiobiological Responses: A Review. Radiat. Res. 178, 505-523 (2012).

33. Connolly, K. A. et al. Increasing the efficacy of radiotherapy by modulating the CCR2/CCR5 chemokine axes. Oncotarget 7, 86522-86535 (2016).

34. Fox, J., Gordon, J. R. \& Haston, C. K. Combined CXCR1/CXCR2 Antagonism Decreases Radiation-Induced Alveolitis in the Mouse. Radiat. Res. 175, 657-664 (2011).

35. Matsumura, S. et al. Radiation-Induced CXCL16 Release by Breast Cancer Cells Attracts Effector T Cells. J. Immunol. 181, 3099-3107 (2008).

36. Yang, X. et al. The Chemokine, CCL3, and Its Receptor, CCR1, Mediate Thoracic RadiationInduced Pulmonary Fibrosis. Am. J. Respir. Cell Mol. Biol. 45, 127-135 (2011).

37. Barker, H. E., Paget, J. T. E., Khan, A. A. \& Harrington, K. J. The tumour microenvironment after radiotherapy: mechanisms of resistance and recurrence. Nat. Rev. Cancer 15, 409-425 (2015).

38. House, I. G. et al. Macrophage-Derived CXCL9 and CXCL10 Are Required for Antitumor Immune Responses Following Immune Checkpoint Blockade. Clin. Cancer Res. 26, 487-504 (2020).

39. Heylmann, D., Rödel, F., Kindler, T. \& Kaina, B. Radiation sensitivity of human and murine peripheral blood lymphocytes, stem and progenitor cells. Biochim. Biophys. Acta BBA - Rev. Cancer 1846, 121-129 (2014).

40. Sia, J., Szmyd, R., Hau, E. \& Gee, H. E. Molecular Mechanisms of Radiation-Induced Cancer Cell Death: A Primer. Front. Cell Dev. Biol. 8, 41 (2020).

41. Heylmann, D., Badura, J., Becker, H., Fahrer, J. \& Kaina, B. Sensitivity of CD3/CD28-stimulated versus non-stimulated lymphocytes to ionizing radiation and genotoxic anticancer drugs: key role of ATM in the differential radiation response. Cell Death Dis. 9, 1053 (2018).

42. Arina, A. et al. Tumor-reprogrammed resident T cells resist radiation to control tumors. Nat. Commun. 10, 3959 (2019).

43. Wirsdörfer, F. \& Jendrossek, V. The Role of Lymphocytes in Radiotherapy-Induced Adverse Late Effects in the Lung. Front. Immunol. 7, (2016). 
44. Qu, Y. et al. 2-Gy whole-body irradiation significantly alters the balance of CD4+CD25-T effector cells and CD4+CD25+Foxp3+T regulatory cells in mice. Cell. Mol. Immunol. 7, 419427 (2010).

45. Berte, N. et al. Impaired DNA repair in mouse monocytes compared to macrophages and precursors. DNA Repair 98, 103037 (2021).

46. Ponath, V. et al. Compromised DNA Repair and Signalling in Human Granulocytes. J. Innate Immun. 11, 74-85 (2019).

47. Leblond, M. M. et al. M2 macrophages are more resistant than M1 macrophages following radiation therapy in the context of glioblastoma. Oncotarget 8, 72597-72612 (2017).

48. Mollà, M. et al. Relative roles of ICAM-1 and VCAM-1 in the pathogenesis of experimental radiation-induced intestinal inflammation. Int. J. Radiat. Oncol. 57, 264-273 (2003).

49. Bezu, L. et al. Combinatorial Strategies for the Induction of Immunogenic Cell Death. Front. Immunol. 6, (2015).

50. Gameiro, S. R. et al. Radiation-induced immunogenic modulation of tumor enhances antigen processing and calreticulin exposure, resulting in enhanced T-cell killing. Oncotarget 5, 403416 (2014).

51. Lotze, M. T. \& Tracey, K. J. High-mobility group box 1 protein (HMGB1): nuclear weapon in the immune arsenal. Nat. Rev. Immunol. 5, 331-342 (2005).

52. Apetoh, L. et al. Toll-like receptor 4-dependent contribution of the immune system to anticancer chemotherapy and radiotherapy. Nat. Med. 13, 1050-1059 (2007).

53. Ghiringhelli, F. et al. Activation of the NLRP3 inflammasome in dendritic cells induces IL-1 $\beta-$ dependent adaptive immunity against tumors. Nat. Med. 15, 1170-1178 (2009).

54. Formenti, S. C. \& Demaria, S. Combining Radiotherapy and Cancer Immunotherapy: A Paradigm Shift. JNCI J. Natl. Cancer Inst. 105, 256-265 (2013).

55. Groves, A. M., Johnston, C. J., Williams, J. P. \& Finkelstein, J. N. Role of Infiltrating Monocytes in the Development of Radiation-Induced Pulmonary Fibrosis. Radiat. Res. 189, 300 (2018).

56. Triner, D. \& Shah, Y. M. Hypoxia-inducible factors: a central link between inflammation and cancer. J. Clin. Invest. 126, 3689-3698 (2016).

57. Martinez-Zubiaurre, I., Chalmers, A. J. \& Hellevik, T. Radiation-Induced Transformation of Immunoregulatory Networks in the Tumor Stroma. Front. Immunol. 9, 1679 (2018).

58. Jobling, M. F. et al. Isoform-Specific Activation of Latent Transforming Growth Factor $\beta$ (LTGF- $\beta$ ) by Reactive Oxygen Species. Radiat. Res. 166, 839-848 (2006).

59. Batlle, E. \& Massagué, J. Transforming Growth Factor- $\beta$ Signaling in Immunity and Cancer. Immunity 50, 924-940 (2019).

60. Costa, A. et al. Fibroblast Heterogeneity and Immunosuppressive Environment in Human Breast Cancer. Cancer Cell 33, 463-479.e10 (2018).

61. Farhood, B. et al. TGF- $\beta$ in radiotherapy: Mechanisms of tumor resistance and normal tissues injury. Pharmacol. Res. 155, 104745 (2020).

62. Liang, H. et al. Host STING-dependent MDSC mobilization drives extrinsic radiation resistance. Nat. Commun. 8, 1736 (2017).

63. Garner, H. \& de Visser, K. E. Immune crosstalk in cancer progression and metastatic spread: a complex conversation. Nat. Rev. Immunol. 20, 483-497 (2020).

64. Mollica Poeta, V., Massara, M., Capucetti, A. \& Bonecchi, R. Chemokines and Chemokine Receptors: New Targets for Cancer Immunotherapy. Front. Immunol. 10, (2019).

65. Henry, C. B. S. \& Duling, B. R. TNF- $\alpha$ increases entry of macromolecules into luminal endothelial cell glycocalyx. Am. J. Physiol.-Heart Circ. Physiol. 279, H2815-H2823 (2000).

66. Zheng, Y., Gao, W., Spratt, D. E., Sun, Y. \& Xing, L. Management of gastrointestinal perforation related to radiation. Int. J. Clin. Oncol. 25, 1010-1015 (2020).

67. Straub, J. M. et al. Radiation-induced fibrosis: mechanisms and implications for therapy. J. Cancer Res. Clin. Oncol. 141, 1985-1994 (2015).

68. Andreyev, J. Gastrointestinal symptoms after pelvic radiotherapy: a new understanding to improve management of symptomatic patients. Lancet Oncol. 8, 1007-1017 (2007). 
69. Henson, C. C. et al. Structured gastroenterological intervention and improved outcome for patients with chronic gastrointestinal symptoms following pelvic radiotherapy. Support. Care Cancer 21, 2255-2265 (2013).

70. Schoenfeld, J. D. et al. Pneumonitis resulting from radiation and immune checkpoint blockade illustrates characteristic clinical, radiologic and circulating biomarker features. J. Immunother. Cancer 7, 112 (2019).

71. Bentzen, S. M. Preventing or reducing late side effects of radiation therapy: radiobiology meets molecular pathology. Nat. Rev. Cancer 6, 702-713 (2006).

72. Nguyen, H. Q. et al. Ionizing radiation-induced cellular senescence promotes tissue fibrosis after radiotherapy. A review. Crit. Rev. Oncol. Hematol. 129, 13-26 (2018).

73. Tabasso, A. F. S., Jones, D. J. L., Jones, G. D. D. \& Macip, S. Radiotherapy-Induced Senescence and its Effects on Responses to Treatment. Clin. Oncol. 31, 283-289 (2019).

74. Langhi Prata, L. G. P., Ovsyannikova, I. G., Tchkonia, T. \& Kirkland, J. L. Senescent cell clearance by the immune system: Emerging therapeutic opportunities. Semin. Immunol. 40, 101275 (2018).

75. Wang, Z., Tang, Y., Tan, Y., Wei, Q. \& Yu, W. Cancer-associated fibroblasts in radiotherapy: challenges and new opportunities. Cell Commun. Signal. 17, 47 (2019).

76. Demaria, S. \& Formenti, S. C. Radiation as an immunological adjuvant: current evidence on dose and fractionation. Front. Oncol. 2, (2012).

77. Rosenstein, B. S. Radiogenomics: Identification of Genomic Predictors for Radiation Toxicity. Semin. Radiat. Oncol. 27, 300-309 (2017).

78. Enaud, R. et al. The Gut-Lung Axis in Health and Respiratory Diseases: A Place for InterOrgan and Inter-Kingdom Crosstalks. Front. Cell. Infect. Microbiol. 10, (2020).

79. Ramírez-Labrada, A. G. et al. The Influence of Lung Microbiota on Lung Carcinogenesis, Immunity, and Immunotherapy. Trends Cancer 6, 86-97 (2020).

80. Meziani, L. et al. CSF1R inhibition prevents radiation pulmonary fibrosis by depletion of interstitial macrophages. Eur. Respir. J. 51, (2018).

81. Wynn, T. A. Fibrotic disease and the TH1/TH2 paradigm. Nat. Rev. Immunol. 4, 583-594 (2004).

82. Dyer, D. P. et al. Chemokine Receptor Redundancy and Specificity Are Context Dependent. Immunity 50, 378-389.e5 (2019).

83. Chung, S. I. et al. IL-13 is a therapeutic target in radiation lung injury. Sci. Rep. 6, 39714 (2016).

84. Wirsdörfer, F. \& Jendrossek, V. Modeling DNA damage-induced pneumopathy in mice: insight from danger signaling cascades. Radiat. Oncol. 12, 142 (2017).

85. Lawrie, T. A. et al. Interventions to reduce acute and late adverse gastrointestinal effects of pelvic radiotherapy for primary pelvic cancers. Cochrane Database Syst. Rev. (2018) doi:10.1002/14651858.CD012529.pub2.

86. Kumagai, T., Rahman, F. \& Smith, A. The Microbiome and Radiation Induced-Bowel Injury: Evidence for Potential Mechanistic Role in Disease Pathogenesis. Nutrients 10, 1405 (2018).

87. Booth, C. \& Potten, C. S. Gut instincts: thoughts on intestinal epithelial stem cells. J. Clin. Invest. 105, 1493-1499 (2000).

88. Malipatlolla, D. K. et al. Long-term mucosal injury and repair in a murine model of pelvic radiotherapy. Sci. Rep. 9, 13803 (2019).

89. Ferreira, M. R. et al. Microbiota-and radiotherapy-induced gastrointestinal side-effects (MARS) study: a large pilot study of the microbiome in acute and late-radiation enteropathy. Clin. Cancer Res. 25, 6487-6500 (2019).

90. Vozenin-Brotons, M.-C. et al. Gene Expression Profile in Human Late Radiation Enteritis Obtained by High-Density cDNA Array Hybridization. Radiat. Res. 161, 299-311 (2004).

91. Blirando, K. et al. Mast Cells Are an Essential Component of Human Radiation Proctitis and Contribute to Experimental Colorectal Damage in Mice. Am. J. Pathol. 178, 640-651 (2011).

92. Dyer, D. P. et al. CXCR2 deficient mice display macrophage-dependent exaggerated acute inflammatory responses. Sci. Rep. 7, 42681 (2017). 
93. Wang, J. et al. Palmitoylethanolamide Regulates Development of Intestinal Radiation Injury in a Mast Cell-Dependent Manner. Dig. Dis. Sci. 59, 2693-2703 (2014).

94. Takemura, N. et al. Eosinophil depletion suppresses radiation-induced small intestinal fibrosis. Sci. Transl. Med. 10, eaan0333 (2018).

95. Kim, H. J. \& Jung, Y. The Emerging Role of Eosinophils as Multifunctional Leukocytes in Health and Disease. Immune Netw. 20, e24 (2020).

96. Mishra, A., Hogan, S. P., Lee, J. J., Foster, P. S. \& Rothenberg, M. E. Fundamental signals that regulate eosinophil homing to the gastrointestinal tract. J. Clin. Invest. 103, 1719-1727 (1999).

97. Grémy, O., Benderitter, M. \& Linard, C. Acute and persisting Th2-like immune response after fractionated colorectal $\gamma$-irradiation. World J. Gastroenterol. 14, 7075 (2008).

98. Wang, J., Zheng, H., Sung, C.-C., Richter, K. K. \& Hauer-Jensen, M. Cellular Sources of Transforming Growth Factor- $\beta$ Isoforms in Early and Chronic Radiation Enteropathy. Am. J. Pathol. 153, 1531-1540 (1998).

99. Stansborough, R. L. et al. Matrix metalloproteinase expression is altered in the small and large intestine following fractionated radiation in vivo. Support. Care Cancer 26, 3873-3882 (2018).

100. Vujaskovic, Z. et al. Radiation-induced hypoxia may perpetuate late normal tissue injury. Int. J. Radiat. Oncol. 50, 851-855 (2001).

101. Toullec, A. et al. HIF-1 $\alpha$ Deletion in the Endothelium, but Not in the Epithelium, Protects From Radiation-Induced Enteritis. Cell. Mol. Gastroenterol. Hepatol. 5, 15-30 (2018).

102. Uribe-Herranz, M. et al. Gut microbiota modulate dendritic cell antigen presentation and radiotherapy-induced antitumor immune response. J. Clin. Invest. 130, 466-479 (2019).

103. Wang, Z. et al. Gut microbial dysbiosis is associated with development and progression of radiation enteritis during pelvic radiotherapy. J. Cell. Mol. Med. 23, 3747-3756 (2019).

104. Anand, S. \& Mande, S. S. Diet, Microbiota and Gut-Lung Connection. Front. Microbiol. 9, 2147 (2018).

105. Dang, A. T. \& Marsland, B. J. Microbes, metabolites, and the gut-lung axis. Mucosal Immunol. 12, 843-850 (2019).

106. Artis, D. Epithelial-cell recognition of commensal bacteria and maintenance of immune homeostasis in the gut. Nat. Rev. Immunol. 8, 411-420 (2008).

107. Gerassy-Vainberg, S. et al. Radiation induces proinflammatory dysbiosis: transmission of inflammatory susceptibility by host cytokine induction. Gut 67, 97-107 (2018).

108. Cui, M. et al. Faecal microbiota transplantation protects against radiation-induced toxicity. EMBO Mol. Med. 9, 448-461 (2017).

109. Bingula, R. et al. Desired Turbulence? Gut-Lung Axis, Immunity, and Lung Cancer. J. Oncol. 2017, 1-15 (2017).

110. Tulic, M. K., Piche, T. \& Verhasselt, V. Lung-gut cross-talk: evidence, mechanisms and implications for the mucosal inflammatory diseases. Clin. Exp. Allergy 46, 519-528 (2016).

111. Wang, J. et al. Respiratory influenza virus infection induces intestinal immune injury via microbiota-mediated Th17 cell-dependent inflammation. J. Exp. Med. 211, 2397-2410 (2014).

112. Shibaki, R., Akamatsu, H., Fujimoto, M., Koh, Y. \& Yamamoto, N. Nivolumab induced radiation recall pneumonitis after two years of radiotherapy. Ann. Oncol. 28, 1404-1405 (2017).

113. Budden, K. F. et al. Emerging pathogenic links between microbiota and the gut-lung axis. Nat. Rev. Microbiol. 15, 55-63 (2017).

114. Dyer, D. P., Salanga, C. L., Volkman, B. F., Kawamura, T. \& Handel, T. M. The dependence of chemokine-glycosaminoglycan interactions on chemokine oligomerization. Glycobiology cwv100 (2015) doi:10.1093/glycob/cwv100.

115. McGhee, J. R. \& Fujihashi, K. Inside the Mucosal Immune System. PLoS Biol. 10, e1001397 (2012).

116. Seong, Y. et al. Trafficking receptor signatures define blood plasmablasts responding to tissue-specific immune challenge. JCI Insight 2, (2017). 
117. Mikhak, Z., Strassner, J. P. \& Luster, A. D. Lung dendritic cells imprint T cell lung homing and promote lung immunity through the chemokine receptor CCR4.J. Exp. Med. 210, 1855-1869 (2013).

118. Ruane, D. et al. Lung dendritic cells induce migration of protective $\mathrm{T}$ cells to the gastrointestinal tract. J. Exp. Med. 210, 1871-1888 (2013).

119. Wang, H. et al. Development of small molecule inhibitors targeting TGF- $\beta$ ligand and receptor: Structures, mechanism, preclinical studies and clinical usage. Eur. J. Med. Chem. 191, 112154 (2020).

120. Ihara, S., Hirata, Y. \& Koike, K. TGF- $\beta$ in inflammatory bowel disease: a key regulator of immune cells, epithelium, and the intestinal microbiota. J. Gastroenterol. 52, 777-787 (2017).

121. Kelly, A. et al. Human monocytes and macrophages regulate immune tolerance via integrin av $\beta 8$-mediated TGF $\beta$ activation. J. Exp. Med. 215, 2725-2736 (2018).

122. Puthawala, K. et al. Inhibition of Integrin $\alpha v \beta 6$, an Activator of Latent Transforming Growth Factor- $\beta$, Prevents Radiation-induced Lung Fibrosis. Am. J. Respir. Crit. Care Med. 177, 8290 (2008).

123. Dyer, D. P. Understanding the mechanisms that facilitate specificity, not redundancy, of chemokine-mediated leukocyte recruitment. Immunology 160, 336-344 (2020).

124. Tree, A. C. et al. Dose-limiting Urinary Toxicity With Pembrolizumab Combined With Weekly Hypofractionated Radiation Therapy in Bladder Cancer. Int. J. Radiat. Oncol. Biol. Phys. 101, 1168-1171 (2018).

125. Bertho, A. et al. Preclinical Model of Stereotactic Ablative Lung Irradiation Using Arc Delivery in the Mouse: Effect of Beam Size Changes and Dose Effect at Constant Collimation. Int. J. Radiat. Oncol. 107, 548-562 (2020).

126. Barsoumian, H. B. et al. Low-dose radiation treatment enhances systemic antitumor immune responses by overcoming the inhibitory stroma. J. Immunother. Cancer 8, e000537 (2020).

127. Symonds, P. \& Jones, G. D. D. FLASH Radiotherapy: The Next Technological Advance in Radiation Therapy? Clin. Oncol. 31, 405-406 (2019).

128. Chuah, S. \& Chew, V. High-dimensional immune-profiling in cancer: implications for immunotherapy. J. Immunother. Cancer 8, e000363 (2020).

129. Al-Shafa, F., Arifin, A. J., Rodrigues, G. B., Palma, D. A. \& Louie, A. V. A Review of Ongoing Trials of Stereotactic Ablative Radiotherapy for Oligometastatic Cancers: Where Will the Evidence Lead? Front. Oncol. 9, (2019).

130. Genard, G., Lucas, S. \& Michiels, C. Reprogramming of Tumor-Associated Macrophages with Anticancer Therapies: Radiotherapy versus Chemo- and Immunotherapies. Front. Immunol. 8, (2017).

131. Colton, M., Cheadle, E. J., Honeychurch, J. \& Illidge, T. M. Reprogramming the tumour microenvironment by radiotherapy: implications for radiotherapy and immunotherapy combinations. Radiat. Oncol. Lond. Engl. 15, 254 (2020).

132. Genard, G. et al. Proton irradiation orchestrates macrophage reprogramming through $\mathrm{NF \kappa B}$ signaling. Cell Death Dis. 9, 1-13 (2018).

133. Harrell, C. R. et al. Molecular mechanisms underlying therapeutic potential of pericytes. J. Biomed. Sci. 25, 21 (2018).

134. Shu, H.-K. G. et al. Inhibition of the CXCL12/CXCR4-Axis as Preventive Therapy for Radiation-Induced Pulmonary Fibrosis. PLoS ONE 8, e79768 (2013).

135. Ma, W. et al. Gut Microbiota Shapes the Efficiency of Cancer Therapy. Front. Microbiol. 10, 1050 (2019).

136. Xu, D. \& Esko, J. D. Demystifying heparan sulfate-protein interactions. Annu. Rev. Biochem. 83, 129-157 (2014).

137. Jaillet, C. et al. Radiation-induced changes in the glycome of endothelial cells with functional consequences. Sci. Rep. 7, 5290 (2017).

138. Bonnans, C., Chou, J. \& Werb, Z. Remodelling the extracellular matrix in development and disease. Nat. Rev. Mol. Cell Biol. 15, 786-801 (2014). 
139. McEntee, C. P., Gunaltay, S. \& Travis, M. A. Regulation of barrier immunity and homeostasis by integrin-mediated transforming growth factor $\beta$ activation. Immunology 160, 139-148 (2020).

140. Egeblad, M. \& Werb, Z. New functions for the matrix metalloproteinases in cancer progression. Nat. Rev. Cancer 2, 161-174 (2002).

141. Stansborough, R. L. et al. Vascular endothelial growth factor (VEGF), transforming growth factor beta (TGF $\beta)$, angiostatin, and endostatin are increased in radiotherapy-induced gastrointestinal toxicity. Int. J. Radiat. Biol. 94, 645-655 (2018). 


\section{Glossary}

\section{Tumour microenvironment (TME)}

Solid tumours are embedded within a collection of stromal cells comprised of immune cells, endothelial cells and fibroblasts, structural components like extracellular matrix as well as signalling components including cytokines, chemokines and growth factors. The main role of the tumour-associated niche is to protect and propagate the tumour, and to promote metastasis.

\section{Abscopal responses}

Systemic immune responses that are triggered following radiotherapy to a local tumour site. Such responses are believed to mainly be propagated by $\mathrm{DC}$ and $\mathrm{CD} 8^{+} \mathrm{T}$ cell responses, and lead to control of secondary tumour at distal tissue sites outside the initial radiotherapy area.

\section{Immune checkpoint inhibitors}

Clinically used monoclonal antibodies targeting specific regulatory immune cell receptors that are important for maintaining self-tolerance and limiting inflammatory responses. Certain cancer cells use these so-called 'checkpoint' immune pathways to evade host immunity. The main immune checkpoint inhibitors used as anti-cancer immunotherapy target the cytotoxic T lymphocyte associated protein 4 (CTLA-4), programmed cell death 1 (PD-1) or its ligand (PD-L1).

\section{RT-induced cell death}

Following exposure to ionising radiation cells can undergo cellular death primarily due to DNA damage. Different types of RT-induced cell death are described for different types of cells and cancers depending on their molecular profile. These include death by necrosis, apoptosis or autophagy. Importantly, each of these types of death has the ability to initiate a cascade of immune signalling following the release of damage-associated molecular patterns.

\section{M1-like or M2-like macrophage phenotypes}

' $\mathrm{M} 1$ ' and ' $\mathrm{M} 2$ ' are classifications historically used to define macrophages activated in vitro as pro-inflammatory (when 'classically' activated with IFNy and LPS) or anti-inflammatory 
(when 'alternatively' activated with IL-4 or IL-10), respectively. However, macrophages in vivo are highly specialized and heterogeneous with regards to their phenotypes and functions, which are continuously shaped by their tissue microenvironment. Therefore, the M1/M2 classification is too simplistic to explain the true nature of macrophages in vivo, although these terms are still often used to indicate whether the macrophages in question are more pro- or anti-inflammatory.

\section{Sterile inflammation}

'Sterile inflammation' is an inflammation induced in the absence of pathogenic threat, instead being triggered by tissue damage and release of danger-associated molecular patterns (DAMPs). Release of DAMPs leads to recruitment of immune cells, which are required to clear damaged cells and initiate the tissue repair. Sometimes, sterile inflammation might become excessive and result in pathology, like in RT-induced damage and breakdown of mucosal barriers.

\section{Mitotic catastrophe and delayed cell senescence}

'Mitotic catastrophe' is a type of RT-induced cell death in which the cell is prematurely entering into mitosis or is trapped in a cell cycle arrest for a prolonged time. The resultant aberrant mitosis or 'delayed senescence' leads to cells that are still metabolically active, but can acquire chromosomal aberrations or a pro-inflammatory phenotype (senescenceassociated secretory phenotype) or even lead to development of secondary cancerous sites, and thus contribute to long-term irradiation toxicity. Some cells trapped in these states will die over time via one of the cellular death pathways (necrosis, apoptosis or autophagy). The full mechanism and extent of deleterious effects following these cellular arrests remain unknown.

\section{NLRP3-dependent inflammasome}

The 'NLRP3-dependent inflammasome' is an important regulator of inflammatory responses and can be triggered by a wide range of danger and inflammatory stimuli. This intracellular multimolecular complex acts via the activation of caspases leading to secretion of pro-inflammatory cytokines IL-1ß and IL-18.

\section{Myeloid-derived suppressor cells (MDSCs)}


Tumours can attract myeloid cells to the tumour microenvironment. These cells often have an immunosuppressive phenotype and provide the tumour with protection from host immunity by hindering anti-tumour effector T cell responses. MDSCs can be of monocytic $\left(\mathrm{CD} 11 \mathrm{~b}^{+} \mathrm{Ly}_{6 \mathrm{C}^{+} \mathrm{Gr}-1^{+}}\right.$in mouse, $\mathrm{CD} 11 \mathrm{~b}^{+} \mathrm{CD} 14^{+} \mathrm{CD} 15^{-} \mathrm{HLA}-\mathrm{DR} \mathrm{R}^{\text {low }}$ in human) or granulocytic/polymorphonuclear $\left(\mathrm{CD} 11 \mathrm{~b}^{+} \mathrm{Ly}_{6 \mathrm{G}}{ }^{+} \mathrm{Gr}-1^{+}\right.$in mouse, CD11 $\mathrm{b}^{+} \mathrm{CD} 14^{-} \mathrm{CD} 15^{+}$in human) phenotype.

\section{Endothelial-to-mesenchymal and epithelial-to-mesenchymal transition (EndoMT and EMT)}

'Endothelial-to-mesenchymal transition' (EndoMT) together with 'epithelial-tomesenchymal transition' (EMT) are two processes that are the drivers of endothelial/epithelial progenitor and mesenchymal stem cell differentiation into myofibroblasts in response to either endothelial or epithelial signals as a part of normal wound healing of these two barrier sites. Both the EndoMT and EMT can be accelerated by RT and TGF $\beta$ and are implicated in the late normal tissue toxicity and fibrosis of irradiated organs. 